\title{
Modelado y resolución del problema del encapsulamiento de combustible nuclear gastado
}

Joaquín Bautista, Luis Batet, Manuel Mateo

Recibido: 10 de Diciembre de 2019

Aceptado: 12 de Febrero de 2020

https://doi.org/10.37610/dyo.v0i71.578

\section{Resumen}

En la gestión de residuos de combustible de las centrales nucleares españolas, está previsto activar los almacenes temporales individualizados (ATI) cuando se alcanza la saturación de la piscina destinada a albergar elementos de combustible nuclear gastado durante el periodo de explotación de la instalación; al finalizar dicho periodo, un ATI puede utilizarse como almacén transitorio a corto o largo plazo hasta que el almacén temporal central (ATC) esté disponible. Los elementos combustibles (EC), gastados y ubicados en la piscina de la central nuclear $(\mathrm{CN})$, se seleccionan para su almacenado en seco en contenedores (cápsulas) atendiendo a una serie de restricciones impuestas a cada ATI por el órgano regulador competente en función de los requerimientos que afectan específicamente a los contenedores utilizados (v.gr. la limitación de la carga térmica o el número de posiciones para elementos combustibles). Las características de estas cápsulas condicionan el tiempo mínimo de enfriamiento del combustible en la piscina de la central nuclear y, por consiguiente, el tiempo necesario para completar el vaciado completo de dicha piscina. En este contexto, presentamos el problema del encapsulamiento en una etapa del combustible nuclear gastado, ofreciendo una formulación y un método de resolución en dos fases. En la primera fase, se utiliza un modelo de Programación lineal entera mixta (MILP-1), cuyo objetivo es minimizar el coste de los contenedores (de diverso tipo) que se requieren para reubicar los elementos disponibles en la piscina de una central nuclear. Para la segunda fase se ha implementado un algoritmo exacto (Algoritmo A1) que, partiendo de una solución de MILP-1, determina asignaciones óptimas de elementos a contenedores regionalizados por limitaciones sobre la carga térmica permitida en cada región. El procedimiento conjunto (MILP-1 más A1) es capaz de resolver óptimamente instancias con 1500 elementos combustibles, 6 tipos de regiones térmicas y 4 tipos de contenedores en tiempos de CPU inferiores a 0.75 segundos, repartidos así: 0.5 segundos para MILP-1 más 0.25 segundos para A1.

\section{Palabras clave}

Programación lineal entera mixta (PLEM); Central nuclear (CN); Elemento combustible nuclear; Gestión de residuos nucleares; Calor de decaimiento; Cápsula multipropósito MPC-32; CN Ascó; Almacén Temporal Individualizado (ATI)

\section{Preliminares}

Los sectores energéticos son fundamentales para la actual actividad humana. La Energía, bajo la óptica de los sistemas productivos, pasa por diversos procesos de generación, transporte y distribución y consumo final

La generación de energía eléctrica se basa en la transformación de otras clases de energía (v.gr. cinética, térmica,

\author{
Joaquín Bautista \\ joaquin.bautista@upc.ed \\ ORCID: 0000-0002-2214-4991 \\ Luis Batet* \\ 1luis.batet@upc.edu \\ ORCID: 0000-0003-1882-6313 \\ Manuel Mateo * \\ manel.mateo@upc.edu \\ ORCID: 0000-0002-3975-9116 \\ * Universitat Politècnica de Catalunya. Avda. Diagonal 647 \\ 08028 Barcelona, España.
}

química, lumínica, solar, eólica, mareomotriz, nuclear, etc.). Cuando dicha generación se realiza con fines industriales, los procesos de transformación se llevan a cabo en instalaciones denominadas centrales eléctricas.

En todas las fases del tratamiento energético aparecen numerosos problemas de optimización, algunos de ellos combinatorios, y de simulación, tanto bajo la óptica tecnológica como la de la gestión de operaciones. Así, en la literatura especializada, podemos encontrar trabajos en el marco de la ingeniería nuclear sobre modelos de simulación del comportamiento de un fluido (temperatura y presión) dentro de un contenedor de combustible nuclear gastado [1]-[2], también encontramos trabajos relacionados con el modelado clásico y resolución de problemas de planificación estática de la distribución de la energía eléctrica [3]-[5], así como otros trabajos, también de planificación, que proponen flexibilizar la distribución de energía en la red eléctrica atendiendo a la emergente variabilidad de la demanda [6].

Las centrales nucleares son sin duda centrales eléctricas que ofrecen un alto rendimiento en el proceso de transfor- 
mación de energía. No obstante, el combustible empleado en ellas, debido a su elevada carga radiológica, requiere una serie de controles de carácter tecnológico que están sujetos a una estricta reglamentación a lo largo de todo su ciclo de vida [7]. Dicho ciclo se inicia con la extracción del mineral de uranio, pasa por la fabricación de pastillas de uranio enriquecido para elementos combustibles, y culmina con la gestión de los residuos radiactivos generados por las centrales nucleares.

En las centrales nucleares, los elementos combustibles nuevos se introducen por carga o recarga en el reactor nuclear donde se "queman" por reacción de fisión en cadena, emitiendo energía calorífica y dando lugar a productos de fisión que constituyen el combustible nuclear gastado.

Los elementos combustibles una vez gastados son extraídos del reactor nuclear y se almacenan temporalmente en una piscina donde permanecen 5 años como mínimo (por requisitos tecnológicos que no legales) hasta completar su proceso de "enfriamiento" para reducir su actividad. En esta primera fase de almacenamiento del combustible en bastidores independientes (racks), dentro de la piscina, aparecen problemas de optimización de la fiabilidad del diseño de bastidores y disposición de elementos ante posibles desplazamientos de los mismos [8]. Además, como la capacidad de la piscina está limitada a un número concreto de elementos, surgen otros problemas de gestión del almacenamiento del combustible nuclear gastado vinculados a dicha limitación [9].

Después de al menos 5 años, los elementos combustibles gastados y enfriados pueden extraerse de la piscina, en una o varias etapas, y ser relocalizados en cápsulas o contenedores que presentan mayor o menor rendimiento en la transferencia de calor del combustible en función de la tecnología incorporada a la cápsula, que puede ser más o menos compleja [10]. Generalmente, tales cápsulas están diseñadas específicamente para el almacenamiento en seco (v.gr. cápsulas MPC: Multi Purpose Canister para 32 o más elementos combustibles).

Una vez encapsulado el combustible gastado, el almacenamiento de las cápsulas puede hacerse transitoriamente en un almacén temporal individualizado (ATI), localizado den- tro del propio emplazamiento de la central nuclear, hasta que se efectúa su traslado definitivo ya sea para ser reprocesado o para ser alojado en un almacén temporal centralizado (ATC) o en un almacén geológico profundo (AGP).

En este marco conceptual, proponemos un procedimiento exacto compuesto de dos fases para minimizar el coste de almacenamiento en seco del máximo número de elementos combustibles enfriados en la piscina, empleando el mínimo número de cápsulas MPC-32 [11]-[12], teniendo en cuenta el contenido de la piscina de la central en una fecha preestablecida para su vaciado completo en una sola etapa. La aplicación del procedimiento se ilustra mediante la instancia ASCÓ\#01_6.0 cuyos datos están inspirados en la central nuclear Ascó.

El resto de este trabajo se estructura de la siguiente forma. En la sección 2 describimos algunos sistemas de almacenamiento en seco del combustible nuclear gastado. La sección 3 está dedicada a la descripción, modelado y resolución del problema del encapsulamiento de combustible nuclear gastado. En la sección 4 ilustramos el procedimiento propuesto mediante un caso de estudio inspirado en una central nuclear real. Finalmente, dedicamos la sección 5 a las conclusiones derivadas de este trabajo y a una breve descripción sobre líneas de trabajo futuras.

\section{Sistemas de almacenamiento en seco}

En España la responsabilidad del desmantelamiento completo de las instalaciones nucleares recae en ENRESA, empresa que es también responsable de la gestión definitiva de los residuos nucleares y radiactivos, siendo una actividad relevante dentro de esta gestión la del almacenamiento temporal en seco del combustible nuclear gastado.

Se puede afirmar que no existe una única solución para tal actividad, aunque en general se ha optado por encapsular los elementos combustibles gastados usando contenedores con diversas funciones y características. Por ejemplo, el sistema de almacenamiento y transporte de combustible gastado en Ascó está formado por distintos componentes para encapsular, transportar, transferir y blindar el combustible nuclear gastado (ver Figura 1). 
Figura 1 Componentes del sistema transportar combustible nuclear gastado. El contenedor HI-TRAC permite transferir la cápsula MPC al HI-STORM (fuentes: CSN y NRC) HI-STORM para almacenar y

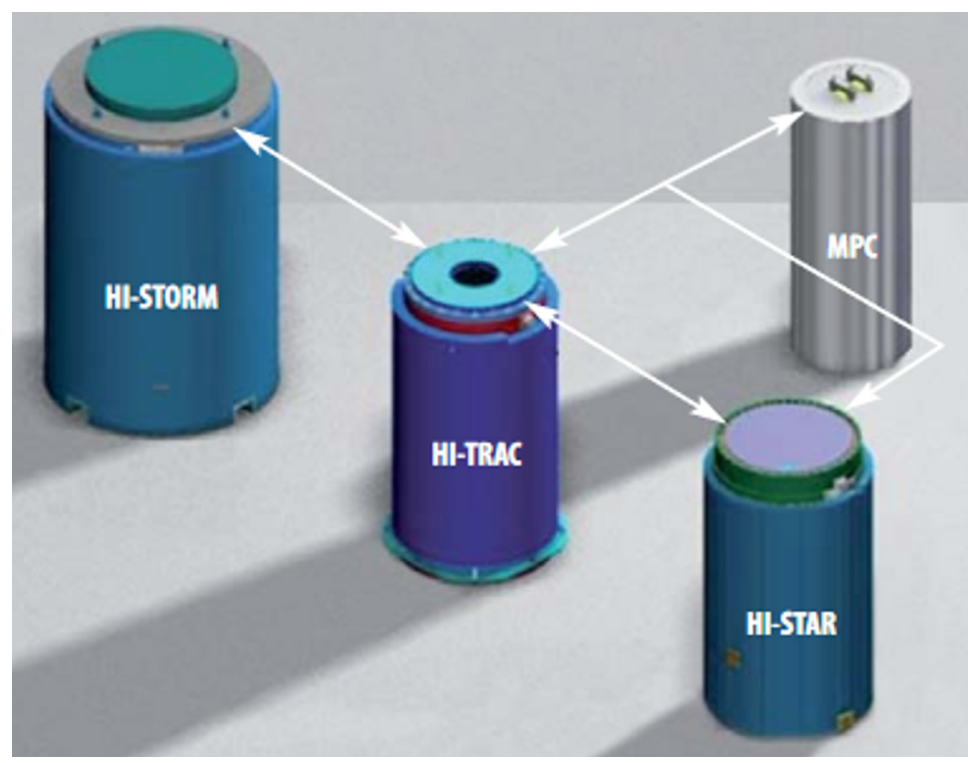

Una solución posible para el almacenamiento es la propuesta HI-STORM que requiere los siguientes componentes: (i) la cápsula multipropósito (MPC-32), (ii) el módulo de almacenamiento (HI-STORM), (iii) el contenedor de transporte (HI-STAR) y (iv) el contenedor de transferencia (HI-TRAC), siendo la función de este último la de transferir los contenidos entre los distintos continentes del sistema de almacenamiento [13].

Atendiendo a la solución de Ascó, para garantizar la integridad del combustible debe limitarse la temperatura a la que pueden verse sometidas las varillas metálicas que envuelven el UO2. Ello, a su vez, impone restricciones en cuanto a la potencia térmica (calor) que pueden generar los elementos combustibles en el interior de la cápsula.
En efecto, en condiciones de carga uniforme cada una de las posiciones de la MPC-32 (ver Figura 2) puede albergar un elemento combustible con una potencia máxima de $0.9375 \mathrm{~kW}$ en el momento de la carga; por tanto, la potencia térmica total disipada por la cápsula será como máximo de $30 \mathrm{~kW}$ ). El sistema permite cargas regionalizadas, de manera que pueden ubicarse en la región central de la cápsula (región permisiva, formada por 12 posiciones) elementos combustibles con una potencia residual mayor, a costa de limitar la potencia máxima por posición en la región periférica (región severa, formada por 20 posiciones) y de limitar también la potencia térmica máxima a un valor inferior o igual a $30 \mathrm{~kW}$. La Figura 2 representa las posiciones asignadas a cada región en una cápsula MPC-32.
Figura 2 Sección transversal de una cápsula multipropósito MPC-32 regionalizada. Las regiones permisiva y severa tienen respectivamente $12 \mathrm{y}$ 20 posiciones para albergar elementos combustibles (fuentes: CSN y NRC)

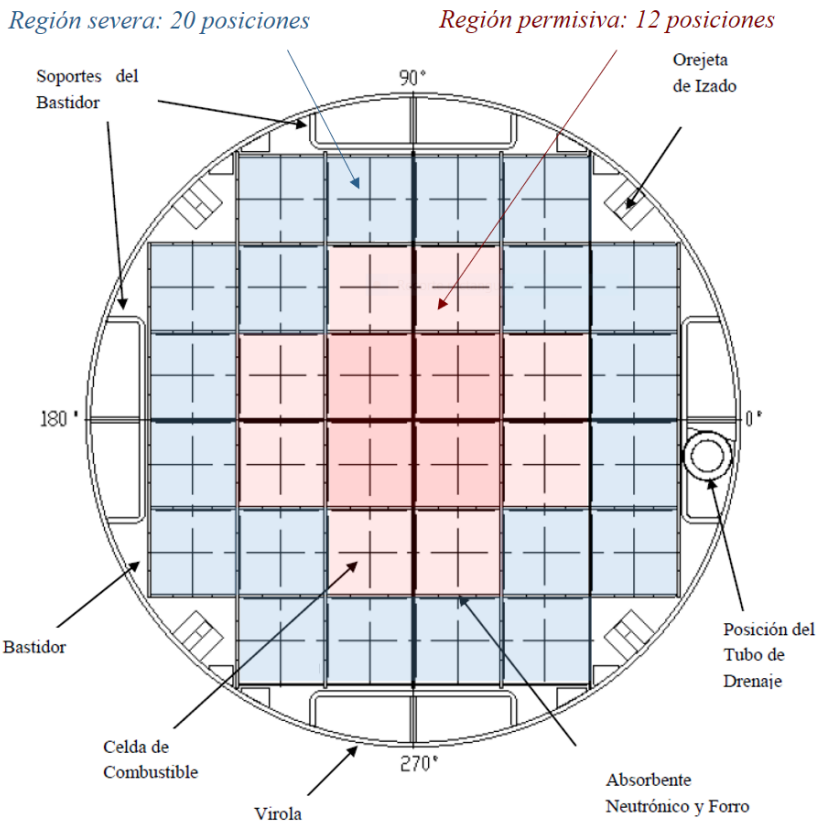




\section{Problema del encapsulamiento de combustible nuclear gastado}

En esta sección trataremos los puntos siguientes: (i) la descripción y formalización del problema que hemos denominado problema del encapsulamiento de combustible nuclear gastado, (ii) la formulación de un modelo matemático basado en la Programación lineal entera mixta (PLEM) para asignar el máximo número de elementos combustibles a regiones caloríficas minimizando el coste de las cápsulas requeridas (Fase 1 del método de resolución) y (iii) la propuesta de un procedimiento de localización concreta de los elementos combustibles en las regiones de un conjunto de contenedores de diverso tipo (Fase 2 del método de resolución).

\subsection{Describiendo el problema}

En un determinado instante $\mathrm{T}$ del horizonte temporal, que corresponde en nuestro caso a la fecha prevista para vaciar en una etapa la piscina de combustible de la central nuclear, se dispone de $n$ elementos de combustible nuclear gastado agrupados en un conjunto $\mathrm{I}(\mathrm{i}=1, ., \mathrm{n}(\mathrm{n} \equiv|\mathrm{I}|))$.

Todo elemento combustible $\mathrm{i} \in \mathrm{I}$ se caracteriza por su potencia térmica o calor residual o de decaimiento [14]-[15] en el instante de extracción $\mathrm{T}$, sea $\mathrm{q}_{\mathrm{i}}(\mathrm{T})$. Tal valor depende del calor residual inicial en el instante de inmersión del elemento $\mathrm{i} \in \mathrm{I}$ en la piscina y del tiempo que este ha permanecido en ella hasta la fecha prevista para el vaciado de la misma.

Por otra parte, se dispone de un sistema de almacenamiento compuesto por un almacén temporal individualizado (ATI) y por un conjunto $\mathrm{K}(\mathrm{k}=0,1, ., \mathrm{l}(|\mathrm{K}| \equiv \mathrm{l}+1))$ de tipos de contenedores de combustible nuclear gastado; donde $\mathrm{k}=0$ simboliza al tipo de contenedor universal capaz de albergar sin restricciones a cualquier elemento combustible, eso sí, a un elevado coste arbitrario $\gamma_{0} \rightarrow \infty$.

A cada tipo de contenedor $(\mathrm{k} \in \mathrm{K})$ se asocia un coeficiente de adversidad $\gamma_{\mathrm{k}}$, cumpliéndose $\gamma_{\mathrm{k}} \ll \gamma_{0} \quad(\forall \mathrm{k} \neq 0)$, el cual corresponde al coste arbitrario por seleccionar un contenedor del tipo $\mathrm{k} \in \mathrm{K}$ para albergar al menos un elemento de combustible nuclear gastado. También supondremos que para el tipo de contenedor $\mathrm{k} \in \mathrm{K}$ se dispone como máximo de un número de unidades igual a $\mathrm{d}_{\mathrm{k}}(\forall \mathrm{k})$, siendo la disponibilidad total de contenedores igual a $D=\sum_{\forall k} d_{k}$.

Los contenedores están regionalizados, es decir, un contenedor está compartimentado en regiones con atributos de capacidad y calor residual. Así, es posible establecer el conjunto de tipos de región de contenedor $\mathrm{J}(\mathrm{j}=0,1, ., \mathrm{m}$
$(|J| \equiv m+1))$ en función de una limitación máxima de calor residual y su capacidad para albergar elementos combustibles; donde $\mathrm{j}=0$ simboliza al tipo de región universal capaz de albergar sin restricciones a cualquier elemento combustible.

Cada tipo de región $(\mathrm{j} \in \mathrm{J})$ se caracteriza por sus límites inferior y superior sobre el calor residual $\left[\mathrm{q}_{\mathrm{j}}, \mathrm{q}_{\mathrm{j}}^{+}\right]$y por su capacidad de almacenamiento $h_{j}$ medida en número de elementos combustibles que como máximo puede albergar un contenedor en dicha región.

La relación entre un tipo de región $(\mathrm{j} \in \mathrm{J})$ y un tipo de contenedor $(\mathrm{k} \in \mathrm{K})$ aquí se establece mediante la matriz $\mathrm{B}$, cuyos coeficientes, $\mathrm{b}_{\mathrm{j}, \mathrm{k}}(\forall \mathrm{j} \forall \mathrm{k})$, adoptan el valor 1 cuando la región de tipo $\mathrm{j} \in \mathrm{J}$ está asociada al contenedor de tipo $\mathrm{k} \in \mathrm{K}$, y valen 0 en caso contrario. Por su parte, la relación entre el elemento combustible $i \in I$ y el tipo de región $j \in J$ se establece a través de la matriz $\mathrm{A}(\mathrm{T})$, cuyos coeficientes, $\mathrm{a}_{\mathrm{i}, \mathrm{j}}(\mathrm{T})(\forall \mathrm{i} \forall \mathrm{j})$, valen 1 cuando el elemento de combustible $i \in I$ puede ser ubicado en la región $\mathrm{j} \in \mathrm{J}$ en el instante $\mathrm{T}$, $\mathrm{y}$ valen 0 en cualquier otro caso; i.e. $a_{i, j}(T)=1 \Leftrightarrow q_{i}(T) \in\left[q_{j} ; q_{j}^{+}\right]$. Obviamente, la relación entre el elemento combustible $\mathrm{i} \in \mathrm{I}$ y el tipo de contenedor $\mathrm{k} \in \mathrm{K}$, queda establecida mediante la matriz $\mathrm{C}(\mathrm{T})$ resultante del producto booleano de las matrices $\mathrm{A}(\mathrm{T})$ y $\mathrm{B}$; esto es, $C(T)=A(T) \otimes B$, cumpliéndose, por tanto, la siguiente propiedad: $\left(\mathrm{a}_{\mathrm{i}, \mathrm{j}}(\mathrm{T})=1 \wedge \mathrm{b}_{\mathrm{j}, \mathrm{k}}=1\right) \Rightarrow \mathrm{c}_{\mathrm{i}, \mathrm{k}}(\mathrm{T})=1$.

En tales condiciones, el problema del encapsulamiento de combustible nuclear gastado (SNFEP: Spent Nuclear Fuel Encapsulation Problem) consiste en transferir el máximo número de los elementos combustibles, ubicados en la piscina de una central nuclear en una fecha $\mathrm{T}$, hacia un conjunto de contenedores regionalizados de diverso tipo, respetando todas las restricciones tecnológicas de capacidad y calor residual y minimizando los costes operativos o, alternativamente, la adversidad global que supone dicha operación.

\subsection{Resolviendo el problema}

Tal como se indicó más arriba, nuestra propuesta resuelve el problema en dos fases. La primera fase emplea la PLEM para asignar con mínimo coste los elementos combustibles a regiones térmicas [16], mientras que para la segunda se ha diseñado una familia de algoritmos exactos para ubicar los elementos en las regiones de los contenedores.

\subsubsection{Fase 1: Modelo PLEM para minimizar el coste de encapsulamiento}

Para formalizar los métodos de resolución propuestos en este trabajo utilizaremos la siguiente nomenclatura. 
Instante o fecha prevista para el vaciado de la piscina de la central nuclear en una sola etapa.

Conjunto de elementos combustibles. Sean los elementos: $\mathrm{i}=1, ., \mathrm{n}(\mathrm{n} \equiv|\mathrm{I}|)$. Corresponde a los elementos de combustible nuclear gastado, los cuales se hallan en la piscina de la central nuclear en el instante $\mathrm{T}$.

Conjunto de tipos de región de contenedor según limitación máxima de calor residual. Sean las regiones: $j=0,1, ., m$ con $|J| \equiv m+1$. Supondremos que existe una región universal, $\mathrm{j}=0$, que no presenta limitación máxima de calor residual para los elementos combustibles; a efectos prácticos, los elementos asignados a la región universal permanecerán en la piscina.

Conjunto de tipos de contenedores de combustible nuclear gastado: $\mathrm{k}=0,1, ., \mathrm{l}$ con $|\mathrm{K}| \equiv \mathrm{l}+1$. Supondremos que existe un tipo de contenedor universal, $\mathrm{k}=0$, que está asociado a la región universal $\mathrm{j}=0$, sin presentar limitación máxima de calor residual para los elementos combustible; a efectos prácticos, los elementos asignados al contenedor universal permanecerán en la piscina. Los contenedores considerados corresponden a cápsulas multipropósito que pueden albergar hasta 32 elementos de combustible como máximo (MPC-32).

Calor residual o de decaimiento del elemento de combustible i $\in$ I en el instante de extracción de la piscina T.

Límite superior del calor residual en la región del tipo jeJ.

Límite inferior del calor residual en la región del tipo jeJ. Aquí, supondremos

$\mathrm{q}_{\mathrm{i}}^{-}=0 \forall \mathrm{j} \in \mathrm{J}$.

Matriz de compatibilidad elemento-región, cuyos coeficientes tecnológicos $\mathrm{a}_{\mathrm{i}, \mathrm{j}}$ adoptan el valor 1 si el elemento de combustible $\mathrm{i} \in \mathrm{I}$ puede ser ubicado en una región del tipo $\mathrm{j} \in \mathrm{J}$ en el instante $\mathrm{T}$, y valen 0 en cualquier otro caso. Formalmente:

$a_{i, j} \equiv a_{i, j}(T)=\left\{\begin{array}{lc}0, & \text { if } q_{i}(T)>q_{j}^{+} \\ 1, & \text { en caso contrario }\end{array}\right\}$

Matriz de compatibilidad región-contendor, cuyos coeficientes tecnológicos $\mathrm{b}_{\mathrm{j}, \mathrm{k}}$ valen 1 cuando la región tipo jeJ está asociada al contenedor tipo $\mathrm{k} \in \mathrm{K}$, y valen 0 en caso contrario.

Matriz de compatibilidad elemento-contendor, definida como $\mathrm{C}(\mathrm{T})=\mathrm{A}(\mathrm{T}) \otimes \mathrm{B}$, cuyos coeficientes tecnológicos $\mathrm{c}_{\mathrm{i} i \mathrm{j}}(\mathrm{T})$ adoptan el valor 1 cuando el elemento $\mathrm{i} \in \mathrm{I}$ puede ser ubicado en un contenedor del tipo $\mathrm{k} \in \mathrm{K}$ en el instante $\mathrm{T}$, y valen 0 en caso contrario. Por tanto, se cumple: $c_{i, k} \equiv c_{i, k}(T)=\left\{\begin{array}{cc}1, & \text { if } \sum_{\forall j \in J} a_{i, j}(T) \otimes b_{j, k}=1 \\ 0, & \text { en caso contrario }\end{array}\right\}$

Capacidad de almacenamiento de una región del tipo jeJ medida en número de elementos combustibles por contenedor que como máximo pueden localizase en dicha región.

Capacidad máxima de almacenamiento de un contenedor del tipo $\mathrm{k} \in \mathrm{K}$ medida en número de elementos combustibles. Se cumple la relación: $\mathrm{H}_{\mathrm{k}}=\sum_{\forall \mathrm{j}} \mathrm{b}_{\mathrm{j}, \mathrm{k}} \times \mathrm{h}_{\mathrm{j}}$.

Número de unidades disponibles de contenedores del tipo $\mathrm{k} \in \mathrm{K}$ en el instante previsto para el vaciado de la piscina ( $\mathrm{T}$ ). La disponibilidad total de contenedores prevista para dicho instante es: $D=\sum_{\forall \mathrm{k}} \mathrm{d}_{\mathrm{k}}$.

Coeficiente de adversidad de un contenedor del tipo $\mathrm{k} \in \mathrm{K}$. El valor $\gamma_{\mathrm{k}}$ simboliza el coste arbitrario por seleccionar un contenedor del tipo $\mathrm{k} \in \mathrm{K}$ para albergar elementos de combustible gastado.

Variable binaria que adopta el valor 1 si el elemento de combustible i $\in \mathrm{I}$ se asigna a una región del tipo jeJ, y vale 0 en caso contrario.

Número de elementos de combustible encapsulados en regiones del tipo $\mathrm{j} \in \mathrm{J}$.

Número de contenedores del tipo $\mathrm{k} \in \mathrm{K}$

Función de adversidad global. Representa el coste global de los contenedores usados para albergar el combustible nuclear gastado en la fecha prevista, $\mathrm{T}$, tras el vaciado de la piscina de la central nuclear. 
Haciendo uso de esta nomenclatura, formulamos el siguiente modelo matemático.

MILP-1: Modelo para la asignación de elementos combustibles a tipos de regiones

$$
\min \Gamma(T)=\sum_{k=0}^{l} \gamma_{k} z_{k}
$$

Sujeto a:

$$
\sum_{i=1}^{n} \sum_{j=0}^{m} x_{i, j}=n
$$

$\sum_{j=0}^{m} a_{i, j} x_{i, j}=1$

$\forall i=1, . ., n$

$y_{j}=\sum_{i=1}^{n} a_{i, j} x_{i, j}$

$\forall j=0,1, ., m$

$$
z_{k} \geq \frac{b_{j, k}}{h_{j}} y_{j}
$$$$
\forall j=0,1, ., m ; \forall k=0,1, ., l
$$

$$
z_{k} \leq d_{k}
$$

$x_{i, j} \in\{0,1\}$

$\forall k=0,1, . ., l$

$y_{j} \in \mathbb{Z}^{+} \cup\{0\}$

$\forall i=1, ., n \forall j=0,1, ., m$

$z_{k} \in \mathbb{Z}^{+} \cup\{0\}$

$\forall j=0,1, ., m$

En el modelo MILP-1, la función objetivo (1) representa la minimización de la función de coste de encapsulamiento o adversidad global, cuyo valor coincide con el número total de contenedores cuando se extraen todos los elementos combustibles de la piscina $\left(\mathrm{z}_{0}=0\right)$ y los coeficiente de adversidad son unitarios para todos los tipos de contenedor $\left(\gamma_{\mathrm{k}}=1\right.$ $\forall \mathrm{k}=1, ., \mathrm{l})$. Las igualdades (2) y (3) garantizan la asignación de todos los elementos combustibles a alguna región, incluida la región universal $\mathrm{j}=0$. Las igualdades (4) sirven para determinar el número de elementos combustibles que deben ser encapsulados en cada tipo de región $\mathrm{j} \in \mathrm{J}$. Las restricciones (5) sirven para acotar inferiormente el número de contenedores de cada tipo $\mathrm{k} \in \mathrm{K}$, mientras que las restricciones (6) se emplean para acotar superiormente tal número en función de la disponibilidad de contenedores. La condición (7) impone que las variables de decisión $\left(\mathrm{x}_{\mathrm{i}, \mathrm{j}}\right)$ sean binarias, y finalmente, las condiciones (8) y (9) fuerzan respectivamente la no negatividad e integridad de

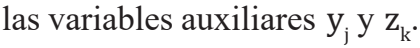

La ejecución del modelo MILP-1 ofrece tres tipos de resultados: (i) una asignación óptima de los elementos combus- tibles a las diversas regiones de contenedor $\left(\mathrm{x}_{\mathrm{i}, \mathrm{j}}{ }^{*} \in\{0,1\} \forall \mathrm{i} \forall \mathrm{j}\right)$, respetando los límites máximos de calor residual permitidos en ellas, (ii) el número óptimo de elementos combustibles que debe albergar cada región $\left(\mathrm{y}_{j}^{*} \forall \mathrm{j}\right)$, y (iii) el número óptimo de contenedores de cada tipo $\left(\mathrm{z}_{\mathrm{k}}^{*} \forall \mathrm{k}\right)$ que corresponde al mínimo coste de encapsulamiento.

Aunque los resultados anteriores son interesantes, queda pendiente concretar la localización de los elementos combustibles en las regiones reales de los contenedores.

\subsubsection{Fase 2: Asignación de los elementos combusibles a las regiones de los contenedores}

Obviamente, existen muchas soluciones para este problema, tantas más cuanto mayores sean los valores de la terna $(n, m, 1)$.

En efecto, si el procedimiento diseñado consiste en asignar, de uno en uno, los elementos combustibles a las regiones concretas dentro de los contenedores, toda ordenación de los objetos correspondientes a las terna de conjuntos (I,J,K), puede dar lugar a una solución distinta. Por ejemplo, si la ordenación recae sobre el conjunto I de elementos combustibles, entonces, el número de formas distintas de asignar es $n$ !, lo cual puede dar lugar a un astronómico número de soluciones potencialmente distintas con instancias de dimensiones realistas, siendo todas ellas igualmente válidas desde el punto de vista de la optimización de la función $\Gamma(\mathrm{T})$.

Atendiendo a esta idea, proponemos una familia de algoritmos constructivos, $\mathrm{A} 1[\pi(\mathrm{n})]$, que es función de la secuencia $\pi(n)$ reguladora de la asignación de los elementos combustibles a las regiones de los contenedores.

En efecto, partiendo de una solución óptima ofrecida por MILP-1 ( $\left.\mathrm{x}_{\mathrm{i}, \mathrm{j}}{ }^{*} \forall \mathrm{i} \forall \mathrm{j}, \mathrm{y}_{\mathrm{j}}{ }^{*} \forall \mathrm{j}, \mathrm{z}_{\mathrm{k}}{ }^{*} \forall \mathrm{k}\right)$, el procedimiento A1[ $\left.\pi(\mathrm{n})\right]$ crea una secuencia $\pi(\mathrm{n})$ de elementos combustibles (sentencia 5 en $A 1[\pi(n)])$ que sirve para establecer el orden de asignación de estos a los contenedores. Una vez determinado el tipo de contenedor $\mathrm{k}_{\mathrm{t}}^{*}$ que corresponde al elemento en curso $\pi_{\mathrm{t}}$, se registra el elemento en la matriz MPC32[•] (sentencia 13 en $A 1[\pi(n)])$, cuyas filas corresponden a contenedores y sus columnas a posiciones dentro del contenedor. Posteriormente, se determinan las cargas de calor residual $\mathrm{Q}_{\mathrm{mpc32}}[\bullet]$ presentes en los contenedores (sentencia 16 en $\mathrm{A} 1[\pi(\mathrm{n})])$ ) y se ordenan los elementos en los contenedores según dichas cargas (sentencia 17 en $\mathrm{A} 1[\pi(\mathrm{n})])$. Finalmente, se muestran los resultados del algoritmo (sentencia 18 en $\mathrm{A} 1[\pi(\mathrm{n})])$ 
$\mathbf{A 1}[\boldsymbol{\pi}(\mathbf{n})]$ : Familia de Algoritmos $\mathrm{A} 1[\pi(\mathrm{n})]$. Procedimiento para asignar elementos combustibles ( $\mathrm{i} \in \mathrm{I}$ ) en el conjunto de contenedores (cápsulas MPC-32). Dada una secuencia $\pi(n)$, el algoritmo retorna una solución constituida por el vector de cargas de calor residual $\left(\mathrm{Q}_{\mathrm{mpc32}}[\bullet]\right)$ presentes en cada contenedor, así como la localización de los elementos combustibles en los contenedores, la cual queda representada por la matriz $\mathrm{MPC} 32[\bullet]$.

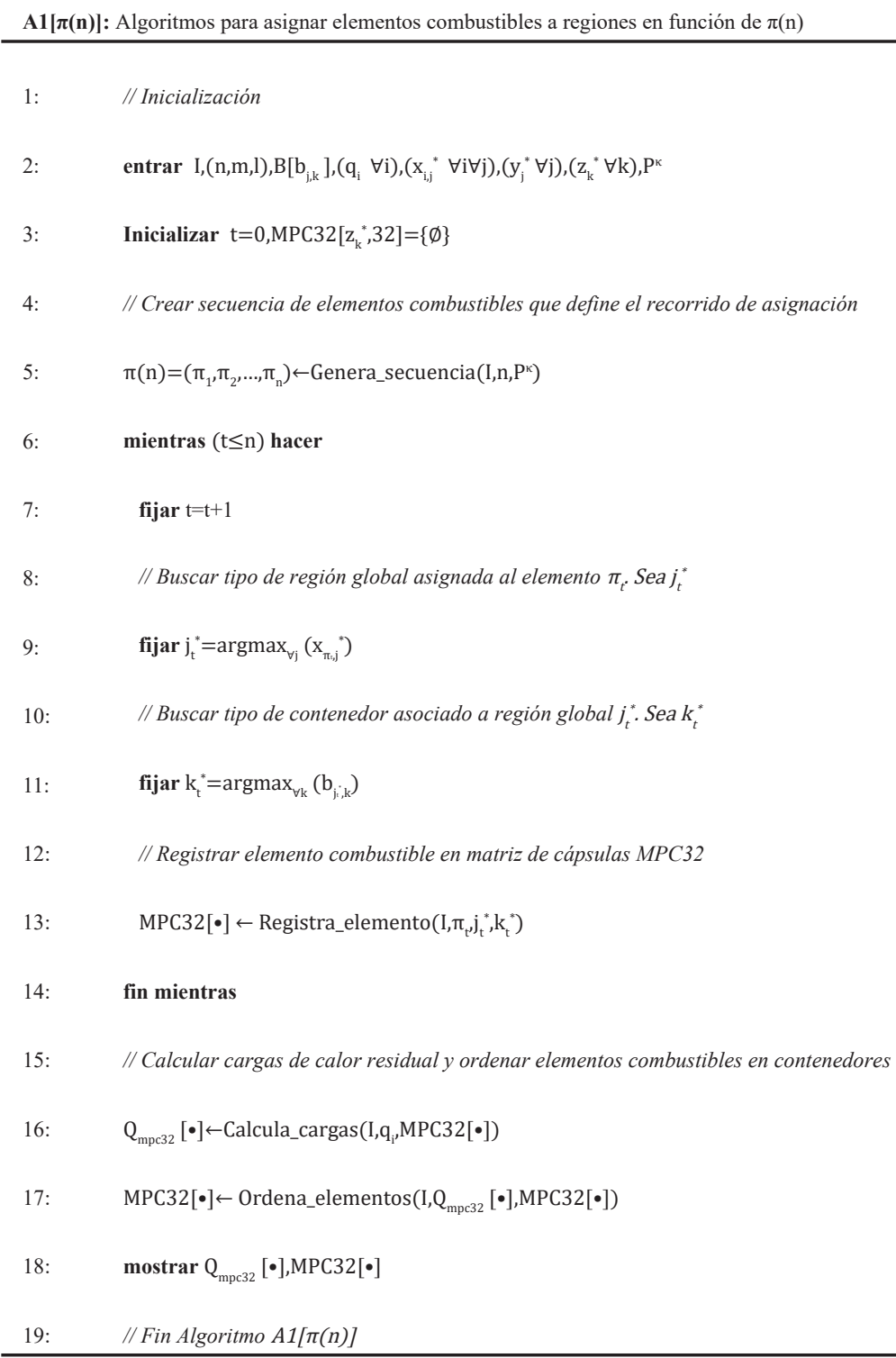

Nótese que $\mathrm{A} 1[\pi(\mathrm{n})]$ ) puede generar multitud de soluciones gracias a que el punto de partida es una permutación $\pi(\mathrm{n})$ de los elementos combustibles del conjunto I (frase 5 en A1 $[\pi(n)]))$. Tales permutaciones se pueden crear utilizando una familia de reglas $\mathrm{P}^{\kappa}$ aplicada sobre el conjunto I que inicialmente está ordenado según el código del elemento. Las reglas $\mathrm{P}^{\kappa}$ pueden ser de diversa naturaleza, pudiendo consistir tanto en la simple ordenación de los elementos según sus calores residuales como en reglas más complejas utilizadas para la generación de vecindarios (intercambio e inserción) en algoritmos basados en trayectorias a través del espacio de búsqueda [17].

Obviamente, todas las soluciones generadas por A1 $[\pi(n)]$ ) son óptimas cuando la función objetivo consiste en mini- mizar el coste de los contenedores, tal como sucede en este trabajo; no obstante, cuando la función objetivo corresponda a otro criterio de optimización, como es el caso del equilibrado de cargas de calor residual en los contenedores, A1 $[\pi(n)]$ ) puede utilizarse al menos de dos formas:

1. En la primera fase de algoritmos multiarranque (v.gr. GRASP) construyendo soluciones iniciales que se utilicen como semillas en la fase posterior de mejora basada en trayectorias en el espacio de búsqueda.

2. En la generación sistemática de soluciones para dar asistencia a metaheurísticas basadas en poblaciones (v.gr. algoritmos genéticos y meméticos). 


\section{Caso de estudio basado en la central nuclear Ascó}

Esta sección está dedicada a un caso de estudio inspirado en la piscina de enfriamiento de elementos combustibles gastados en la central nuclear Ascó y contiene los puntos siguientes: (i) las hipótesis del modelo, (ii) los requisitos para el encapsulamiento del combustible gastado, (iii) los procedimientos empleados, (iv) los datos utilizados y (v) los resultados conseguidos.

\subsection{Hipótesis para el caso}

Se consideran las siguientes hipótesis para el caso que nos ocupa:

H1. Los elementos combustibles están caracterizados por su tipo, grado de quemado (Burnup: $\mathrm{MWd} / \mathrm{t}$ ), enriquecimiento inicial (\% en masa) y la fecha de extracción del núcleo según el último ciclo de quemado, lo cual se traduce en el tiempo que transcurre desde su última descarga del núcleo medido en años. A partir de estos datos, puede determinarse el calor residual de cada elemento en el instante de extracción de la piscina.

H2. No se consideran los elementos combustibles dañados ni los que puedan estar afectados por otras restricciones adicionales.

H3. No se consideran en el modelo los elementos asociados al combustible, tales como fuentes neutrónicas, barras de control, venenos consumibles y otros.

H4. Se supone que sólo se dispone de un tipo de cápsula en cuanto a dimensiones espaciales físicas y número de posiciones para los elementos combustibles. La cápsula que se utiliza en este caso es del tipo MPC-32 y el contenedor que actúa de blindaje biológico durante algunas fases del proceso de carga es el HI-TRAC (transferencia).

H5. Los elementos combustibles se cargan en contenedores teniendo en cuenta los requisitos de almacenado en módulos tipo HI-STORM (contenedor de almacenamiento); se supone, además, que no hay limitación de capacidad en la losa de hormigón del almacén temporal individualizado (ATI).

H6. Se pretende el vaciado total de la piscina, si ello es posible, en una fecha determinada que puede corresponder a la del cese de operaciones en la central nuclear. Se consideran sólo los elementos presentes en la piscina en ese momento, es decir, no se ha considerado que en el caso de cese de operaciones el contenido del último núcleo se descargaría en la piscina y se debería contar con 157 elementos combustibles adicionales con diver- sos grados de quemado. El contenido de la piscina que se ha considerado en el estudio no es el actual, sino que corresponde a una situación anterior en Ascó.

H7. El vaciado de la piscina se debe realizar con el mínimo número de contenedores en la fecha más temprana posible. Se supone que la carga de los contenedores es instantánea, por lo que no es necesario cargar ningún contenedor antes de transcurridos 5 años tras la entrada en piscina del último elemento combustible.

\subsection{Requisitos para el encapsulamiento de elementos combustibles}

Los requisitos que se han tenido en cuenta para el vaciado de la piscina son estos:

R.1. Los contenedores para los elementos combustibles son del tipo MPC-32, los cuales están compartimentados en 32 posiciones, pudiendo contener cada una un solo elemento (ver Figura 2).

R.2. Las posiciones de un contenedor se agrupan en dos regiones: $\left(\mathrm{R}_{\mathrm{a}}\right)$ región permisiva con alto límite de calor de decaimiento que está constituida por $\mathrm{h}_{\mathrm{a}}=12$ posiciones y $\left(\mathrm{R}_{\mathrm{b}}\right)$ región severa con bajo límite de calor de decaimiento constituida por $\mathrm{h}_{\mathrm{b}}=20$ posiciones.

R.3. Una posición de encapsulamiento de combustible (concepto para el que emplearemos el sustantivo latino locus-loci) puede albergar un elemento cuyo tiempo de enfriamiento debe ser mayor o igual al mínimo permitido, el cual corresponde a 5 años desde su extracción del núcleo del reactor por requisitos tecnológicos.

R.4. El contenido de cada locus en todo contenedor debe poseer un calor de decaimiento inferior o igual al máximo permitido en el locus: $\mathrm{q}_{\mathrm{a}}^{+}$en la región $\mathrm{R}_{\mathrm{a}} \mathrm{y} \mathrm{q}_{\mathrm{b}}^{+}$ en la región $R_{b}$.

R.5. El máximo calor residual permitido en toda cápsula $\mathrm{MPC}-32$ es $\mathrm{Q}_{\mathrm{MPC}}{ }^{+}=30 \mathrm{~kW}$.

R.6. Los máximos valores tolerados para el calor de decaimiento en los loci se determinan según sea el caso:

Caso de carga uniforme: el máximo calor de decaimiento permitido por locus de la cápsula MPC-32 es igual a $\mathrm{q}_{\mathrm{a}}^{+}=\mathrm{q}_{\mathrm{b}}^{+}=0,9375 \mathrm{~kW}(30 \mathrm{~kW} / 32)$. Por tanto, si todos los elementos combustibles presentan un calor de decaimiento inferior o igual al límite de carga uniforme (i.e. $\mathrm{q}_{\mathrm{i}}(\mathrm{T}) \leq 0,9375 \mathrm{~kW}$ $\forall i \in I$ ), entonces, se empleará un solo tipo de contenedor y el problema tiene solución trivial. 
- Caso de carga regionalizada: Cuando no es posible vaciar completamente la piscina empleando solo contenedores de carga uniforme, se utiliza la carga regionalizada [18]. Para determinar los valores máximos de calor de decaimiento por locus $\left(\mathrm{q}_{\mathrm{a}}^{+} \mathrm{o}\right.$ $\mathrm{q}_{\mathrm{b}}^{+}$) según la región a la que pertenece $\left(\mathrm{R}_{\mathrm{a}} \mathrm{o} \mathrm{R}_{\mathrm{b}}\right)$, se aplica el procedimiento que sigue:

(1) Fijar un valor de la ratio de cargas térmicas máximas $\rho=\left(q_{a}^{+}\right) /\left(q_{b}^{+}\right)$, tal que: $1 \leq \rho \leq 3$.

(2) Determinar la carga térmica máxima, $\mathrm{q}_{\mathrm{b}}{ }^{+}=\mathrm{q}_{\mathrm{b}}{ }^{+}$ $\left(\rho, h_{a}, h_{b}\right)$, para la región severa $R_{b}$, a partir de la expresión:

$\left(\mathrm{h}_{\mathrm{a}} \times \rho+\mathrm{h}_{\mathrm{b}}\right) \times \mathrm{q}_{0}{ }^{+}\left(\rho, \mathrm{h}_{\mathrm{a}}, \mathrm{h}_{\mathrm{b}}\right)=\mathrm{f}\left(\rho, \mathrm{h}_{\mathrm{a}^{\prime}} \mathrm{h}_{\mathrm{b}}\right) \times \mathrm{Q}_{\mathrm{MPC}}{ }^{+}$

Siendo $\mathrm{f}\left(\rho, \mathrm{h}_{\mathrm{a}}, \mathrm{h}_{\mathrm{b}}\right)$ una función acotada a 1 para $\rho=1$ y decreciente con $\rho$, de manera que la carga térmica está penalizada por utilizar la opción de carga regionalizada. La función $\mathrm{f}\left(\rho, \mathrm{h}_{\mathrm{a}}, \mathrm{h}_{\mathrm{b}}\right)$ para la cápsula MPC-32 es la siguiente:

$$
f\left(\rho, h_{a}, h_{b}\right)=\frac{2}{\left(1+\rho^{\left(0.23 / \rho^{0.1}\right)}\right)}
$$

(3) Determinar la carga térmica máxima, $\mathrm{q}_{a}^{+}$ $\left(\rho, h_{a}, h_{b}\right)$, para la región permisiva $R_{a}$ :

$$
\mathrm{q}_{\mathrm{a}}^{+}\left(\rho, \mathrm{h}_{\mathrm{a}}, \mathrm{h}_{\mathrm{b}}\right)=\mathrm{q}_{\mathrm{b}}^{+}\left(\rho, \mathrm{h}_{\mathrm{a}^{\prime}} \mathrm{h}_{\mathrm{b}}\right) \times \rho
$$

\subsection{Datos y otras características del caso}

Obviamente, la dimensión del modelo MILP-1 es función de la instancia empleada. En este trabajo utilizamos la instancia Ascó\#01_6.0 (ver Tabla A1 en Anexo I) como juego de datos de calores de decaimiento. Las características principales del experimento son las que siguen:

(1) El número de elementos combustibles gastados es igual a $n=1164$. Los elementos se identifican con los índices $\mathrm{i} \in \mathrm{I}$ correspondientes a los n primeros números naturales.

(2) La fecha prevista para la extracción de elementos de la piscina es $\mathrm{T}=6$ años desde el momento de cese de la actividad. Los calores de decaimiento $\mathrm{q}_{\mathrm{i}}(\mathrm{T}) \quad \forall \mathrm{i} \in \mathrm{I}$ listados en la tabla A1 del Anexo I corresponden a $\mathrm{T}=6$ años.

(3) El número de tipos de región virtual de calor residual por posición, $\mathrm{q}$ en $\mathrm{kW}$, es igual a $\mathrm{m}=6$. Se consideran 6 regiones (sean: $\mathrm{R}_{\mathrm{j}}=\left\{\mathrm{q} \in \mathrm{R}: \mathrm{q} \in\left[\mathrm{q}_{\mathrm{j}}{ }^{-} \mathrm{q}_{\mathrm{j}}^{+}\right]\right\} \quad \forall \mathrm{j}=0,1, ., 5$ ), las cuales se definen con intervalos cerrados de calor residual por locus. Las regiones se concretan a partir de tres valores de la ratio de cargas térmicas máximas: $\rho=1,2,3$. Sean:
$R_{0}=\{q \in R: q \in[0.00,30]\}$

$R_{1}=\{q \in R: q \in[0.00,1.4250]\}$

$R_{2}=\{q \in R: q \in[0.00,1.2620]\}$

$R_{3}=\{q \in R: q \in[0.00,0.9375]\}$

$R_{4}=\{q \in R: q \in[0.00,0.6310]\}$

$R_{5}=\{q \in R: q \in[0.00,0.4750]\}$

Por comodidad, las regiones virtuales han sido numeradas según el orden decreciente del límite superior $\mathrm{q}_{j}^{+} \forall \mathrm{j}$ de cada intervalo.

(4) La definición de tipos de región virtual $\left(\mathrm{R}_{0} \mathrm{a} \mathrm{R}_{5}\right)$ permite establecer la matriz de compatibilidad elemento-región $\mathrm{A}(\mathrm{T})$, cuyos coeficientes tecnológicos adoptan el valor 1 si el elemento de combustible $i \in I$ puede ser asignado a una región virtual del tipo $\mathrm{j} \in \mathrm{J}$ en el instante $\mathrm{T}$, $\mathrm{y}$ valen 0 en cualquier otro caso. Formalmente:

$$
\begin{gathered}
a_{i, j} \equiv a_{i, j}(T=6)=\left\{\begin{array}{ll}
0, & \text { if } q_{i}(T=6)>q_{j}^{+} \\
1, & \text { en caso contrario }
\end{array}\right\} \\
\forall i=1, \ldots, 1164 ; \forall j=0,1, \ldots, 5
\end{gathered}
$$

(5) El número de tipos de contenedor es $1=3$ cuando prescindimos de la región virtual neutra $R_{0}$ que corresponde a la piscina. La correspondencia entre las regiones térmicas $\left(R_{1} a R_{5}\right)$ y los tipos reales de contenedor regionalizado $\left(\mathrm{CR}_{1}\right.$ a $\left.\mathrm{CR}_{3}\right)$ se establece a través de la matriz $\mathrm{B}$ de compatibilidad regióncontenedor, cuyos coeficientes $b_{\mathrm{j}, \mathrm{k}}$ valen 1 cuando la región $R_{j}(j=1, ., 5)$ está asociada al contenedor $C_{k}$ $(\mathrm{k}=1, ., 3)$, y valen 0 en caso contrario. En nuestro caso, la matriz $\mathrm{B}$ es la que sigue:

$$
B_{m \times l}=B_{5 \times 3}=\left(\begin{array}{ccc}
1 & 0 & 0 \\
0 & 1 & 0 \\
0 & 0 & 1 \\
0 & 1 & 0 \\
1 & 0 & 0
\end{array}\right)
$$

Así, un contenedor $\mathrm{CR}_{1}$ está compartimentado en una región permisiva con las características de $\mathrm{R}_{1}$ y una región severa asociada a $\mathrm{R}_{5}$; un contenedor $\mathrm{CR}_{2}$ está asociado a una región permisiva $R_{2} y$ a una severa $R_{4}$; $y$, finalmente, un contenedor tipo $\mathrm{CR}_{3}$ posee una sola región con las cualidades de la región intermedia $R_{3}$.

(6) Desde el punto de vista tecnológico, todos los contenedores de este estudio corresponden a cápsulas MPC-32 (multi-purpose canister con 32 loci). Las 32 posiciones de una MPC-32 se reparten entre las regiones físicas de calor residual de la forma siguiente: 12 posiciones para la región permisiva y 20 para la severa. Obviamente, una MPC-32 tipo $\mathrm{CR}_{3}$ posee 32 posiciones con el mismo límite superior para el calor residual: $\mathrm{q}_{3}{ }^{+}=0.9375$ $\mathrm{kW}$ por locus (30kW por cápsula). 
(7) Teniendo en cuenta que el número de tipos de contenedor es $1=3$, queda por definir el coste por uso de cada tipo de contenedor. Para medir el impacto sobre las soluciones que ofrece MILP-1 en función de los coeficientes de adversidad $\gamma_{\mathrm{k}}(\forall \mathrm{k})$, daremos valores distintos a la terna de costes arbitrarios $\vec{\gamma}=\left(\gamma_{1}, \gamma_{2}, \gamma_{3}\right)$.

(8) Atendiendo a los valores y características expuestos en los puntos anteriores, las dimensiones del modelo MILP-1 para la instancia Ascó\#01_6.0 son las que siguen: (i) 6984 variables de decisión binarias $\left(\mathrm{x}_{\mathrm{i}, \mathrm{j}}\right)$ correspondientes a la asignación de elementos combustibles a regiones térmicas virtuales; (ii) 6 variables enteras $\left(\mathrm{y}_{\mathrm{j}}\right)$ asociadas al número de elementos combustibles albergados por cada región térmica virtual; (iii) 4 variables enteras $\left(\mathrm{z}_{\mathrm{k}}\right)$ representativas del número de contenedores de cada tipo; y (iv) 1195 restricciones o, bien, 1199 restricciones cuando se acota superiormente la disponibilidad de contenedores.

\subsection{Procedimientos}

Todos los códigos compilados correspondientes a los procedimientos utilizados han sido ejecutados en un ordenador DELL Inspiron-13 (Intel(R) Core(TM) i7-7500U@2.70 GHz CPU 2.90 GHz, 16 GB de RAM, x64 Windows 10 Pro).

Los modelos y herramientas utilizados para hallar las soluciones óptimas para el caso que nos ocupa son los que siguen:

(1) Modelo MILP-1 basado en PLEM que permite minimizar el coste $\left(\Gamma^{*}(\mathrm{~T})\right)$ de los contenedores requeridos $\left(\mathrm{z}_{\mathrm{k}}{ }^{*} \forall \mathrm{k}\right)$ para encapsular el combustible nuclear gastado que se halla en la piscina de la central nuclear. MILP-1 ofrece además el número de elementos contenidos en cada región $\left(\mathrm{y}_{\mathrm{i}}^{*} \forall \mathrm{j}\right)$, así como una asignación óptima $\left(\mathrm{x}_{\mathrm{i}, \mathrm{j}}{ }^{*} \forall \mathrm{i} \forall \mathrm{j}\right)$ de los elementos combustibles a tipos de regiones de calor de decaimiento. Para la explotación de MILP-1 se ha utilizado el solucionador IBM ILOG CPLEX (Optimization Studio v.12.2, win-x86-64).

(2) Método exacto $\mathrm{A} 1[\pi(\mathrm{n})]$ que parte del resultado de MILP-1 y ofrece una asignación de los elementos combustibles a las regiones permisivas y severas de los contenedores.
En definitiva, a partir de una solución óptima hallada por MILP-1, la cual queda caracterizada por la terna de conjuntos de valores $\left[\left(\mathrm{x}_{\mathrm{i}, \mathrm{j}}{ }^{*} \forall \mathrm{i} \forall \mathrm{j}\right),\left(\mathrm{y}_{\mathrm{j}}{ }^{*} \forall \mathrm{j}\right),\left(\mathrm{z}_{\mathrm{k}}{ }^{*} \forall \mathrm{k}\right)\right]$, se aplica el método exacto $A 1[\pi(n)]$, el cual transfiere, de uno en uno y siguiendo una secuencia $\pi(\mathrm{n})$ según la regla $\mathrm{P}^{\kappa}$, los elementos combustibles localizados en las regiones virtuales $R_{i}(\forall j)$ hacia las regiones físicas (permisiva $y$ severa) de los contenedores $\mathrm{CR}_{\mathrm{k}}(\forall \mathrm{k})$, utilizando la matriz $\mathrm{B}$ de compatibilidad región-contenedor.

\subsection{Resultados}

Para medir el impacto sobre las soluciones que ofrece MILP-1 en función de los coeficientes de adversidad $\gamma_{\mathrm{k}}(\forall \mathrm{k})$, se analizan 7 soluciones óptimas que corresponde a ejecutar MILP-1 fijando la terna de costes arbitrarios $\vec{\gamma}=\left(\gamma_{1}, \gamma_{2}, \gamma_{3}\right)$ a los valores siguientes:

$$
\begin{aligned}
& \vec{\gamma}_{0}=(1,1,1), \vec{\gamma}_{1}=(3,2,1), \vec{\gamma}_{2}=(3,1,2), \vec{\gamma}_{3}=(2,3,1) \\
& \vec{\gamma}_{4}=(2,1,3), \vec{\gamma}_{5}=(1,3,2), \vec{\gamma}_{6}=(1,2,3)
\end{aligned}
$$

Tras hallar cada solución óptima mediante MILP-1 $\left[\left(\mathrm{x}_{\mathrm{i}, j}{ }^{*}\right.\right.$ $\left.\forall \mathrm{i} \forall \mathrm{j}),\left(\mathrm{y}_{\mathrm{i}}^{*} \forall \mathrm{j}\right),\left(\mathrm{z}_{\mathrm{k}}{ }^{*} \forall \mathrm{k}\right)\right]$, se aplica el Algoritmo A1[ $\left.\pi(\mathrm{n})\right]$ utilizando la secuencia de asignación $\pi(\mathrm{n})$ correspondiente al orden lexicográfico de los códigos de los elementos combustibles.

Los resultados globales de las 7 soluciones se recogen en la Tabla 1. Por otra parte, los resultados detallados de dichas soluciones óptimas, según $\vec{\gamma}=\left(\gamma_{1}, \gamma_{2}, \gamma_{3}\right)$, están disponibles en el Anexo I de la referencia [19].

Concretamente, en la Tabla 1 se recogen para cada solución (\#0 a \#6) los siguientes datos: (i) el coste imputado a cada tipo de contenedor $\left(\vec{\gamma}=\left(\gamma_{1}, \gamma_{2}, \gamma_{3}\right)\right)$, (ii) el número óptimo de contenedores de cada tipo $\left(\mathrm{z}_{\mathrm{k}}{ }^{*} \forall \mathrm{k}\right)$ y el número total $\left(\mathrm{z}_{\text {tot }}{ }^{*}\right) \mathrm{de}$ contenedores requeridos por cada solución, (iii) los valores óptimos de la función coste objetivo del modelo MILP-1 $\left(\Gamma^{*}(\mathrm{~T})\right)$, (iv) el calor residual medio correspondiente a la configuración de cada solución $\left(\mathrm{Q}_{\mathrm{med}}\right)$, y (v) la desviación tipo del calor residual en el conjunto de cápsulas tipo $(\sigma(\mathrm{Q}))$. 
Tabla 1 Resumen de resultados de las 7 soluciones óptimas (\#0 a \#6) para la instancia Ascó\#01_06 con las siguientes características técnicas: (i) 1164 elementos de combustible nuclear gastado, (ii) 6 años de cese de la actividad de los elementos, (iii) 6 regiones térmicas (incluida la región neutra) definidas por tres valores de la ratio de cargas térmicas máximas $\rho$ (1, 2 y 3), y (iv) 4 tipos de contenedor incluido el contenedor neutro.

\begin{tabular}{|c|c|c|c|c|c|c|c|c|c|c|}
\hline Solución \# & $\gamma_{1}$ & $\gamma_{2}$ & $\gamma_{3}$ & $\mathrm{z}_{1}{ }^{*}$ & $\mathbf{z}_{2}{ }^{*}$ & $\mathbf{z}_{3}{ }^{*}$ & $\mathbf{z}_{\text {tot }}^{*}$ & $\Gamma^{*}(\mathrm{~T})$ & $\mathbf{Q}_{\text {med }}$ & $\sigma(Q)$ \\
\hline$\# 0$ & 1 & 1 & 1 & 4 & 17 & 16 & 37 & 37 & 22.7683 & 3.349247 \\
\hline$\# 1$ & 3 & 2 & 1 & 2 & 17 & 18 & 37 & 58 & 22.7683 & 3.287297 \\
\hline$\# 2$ & 3 & 1 & 2 & 2 & 23 & 12 & 37 & 53 & 22.7683 & 3.001163 \\
\hline$\# 3$ & 2 & 3 & 1 & 19 & 0 & 24 & 43 & 62 & 19.5914 & 4.421877 \\
\hline$\# 4$ & 2 & 1 & 3 & 2 & 54 & 0 & 56 & 58 & 15.0434 & 4.549144 \\
\hline$\# 5$ & 1 & 3 & 2 & 19 & 0 & 24 & 43 & 67 & 19.5914 & 4.605397 \\
\hline \#6 & 1 & 2 & 3 & 40 & 16 & 0 & 56 & 72 & 15.0434 & 4.100421 \\
\hline
\end{tabular}

En la Tabla 1 aparecen 3 soluciones óptimas que requieren 37 cápsulas, número que además coincide con el mínimo número de contenedores según la cota inferior ([1164/32]), no obstante, dichas soluciones presentan diferentes valores de la desviación tipo de la carga térmica en función de la configuración de las 37 cápsulas.

A modo de ejemplo, la solución \#2, que es la que presenta menor dispersión de carga térmica entre las cápsulas, corresponde a la terna de costes $\vec{\gamma}_{2}=(3,1,2)$, en la cual se considera que la adversidad al uso de los contenedo- res tipo 1 y 3 triplica y duplica, respectivamente, la de los contenedores de tipo 2. La solución \#2 detallada se recoge en el Anexo II de este trabajo.

Atendiendo a la solución \#2, la columna ECs de la Figura 3 muestra el número de elementos asignados a cada región ficticia, i.e. $\mathrm{y}_{1}{ }^{*}=24, \mathrm{y}_{2}{ }^{*}=274, \mathrm{y}_{3}{ }^{*}=369, \mathrm{y}_{4}{ }^{*}=457, \mathrm{y}_{5}{ }^{*}=40$. Esto se traduce en un requerimiento total de 37 cápsulas MPC-32 (ver columna $\operatorname{MPC}\left[\mathrm{z}_{\mathrm{k}}, \mathrm{h}_{\mathrm{j}}\right]$ de la Figura 3 ), cuyo reparto entre sus tipos 1,2 y 3 corresponde a 2, 23 y 12 contenedores regionalizados, respectivamente.
Figura 3 Solución \#2: número de elementos combustibles y de contenedores de cada región térmica

\begin{tabular}{|c|c|c|c|c|c|c|c|}
\hline Zona & Limites & Q-Zona & $\mathrm{ECs}$ & MPC -32 & Limites Q-Región & \multicolumn{2}{|c|}{$\operatorname{MPC}\left[\mathrm{z}_{\mathrm{k}}, \mathrm{h}_{j}\right]$} \\
\hline Zona 1 & {$[1.2620$} & 1. 4250$]$ & 24 & T1-RA & {$[0.0000,1.4250]$} & 2 & (12) \\
\hline Zona 2 & {$[0.9375$} & 1. 2620$]$ & 274 & T2-RA & {$[0.0000,1.2620]$} & 23 & (12) \\
\hline Zona 3 & {$[0.6310$} & $0.9375]$ & 369 & T3-RAB & {$[0.0000,0.9375]$} & 12 & (32) \\
\hline Zona 4 & {$[0.4750$} & $0.6310]$ & 457 & $\mathrm{~T} 2-\mathrm{RB}$ & {$[0.0000,0.6310]$} & 23 & (20) \\
\hline Zona 5 & {$[0.0000$} & $0.4750]$ & 40 & $\mathrm{~T} 1-\mathrm{RB}$ & {$[0.0000,0.4750]$} & 2 & (20) \\
\hline Total & lemen & E( & 1164 & Total & cápsulas MPC-32 & 37 & (32) \\
\hline
\end{tabular}

Por su parte, en la Figura 4 se muestra la carga detallada de la MPC-32 número 24 de la solución \#2 (ver Anexo II). Dicha carga se caracteriza por los códigos de los elementos combustibles y por los calores residuales $(\mathrm{kW})$ de estos.

En la Figura 4 podemos ver que la cápsula 24 de la solución \#2 es del tipo 2 con una región permisiva $R_{a} \leftarrow R_{2}$ de 12 posiciones y una región severa $R_{b} \leftarrow R_{4}$ de 20. En la región $\mathrm{R}_{\mathrm{a}}$ se han localizado los 12 elementos más calientes de esta cápsula, de manera que el locus-1 está ocupado por el elemento combustible 827 con un calor residual de 1.2575 $\mathrm{kW}$, mientras que en locus-12 (último de $\mathrm{R}_{\mathrm{a}}$ ) está localizado el elemento combustible 829 con un calor residual de 1.0519 $\mathrm{kW}$. Por su parte, en la región severa $\mathrm{R}_{\mathrm{b}}$ de la cápsula 24 están ubicados 20 elementos fríos en las posiciones 13 a 32, ocupando el elemento 1077 el locus-13 y el elemento 1068 el locus-32, siendo sus calores de decaimiento iguales a 0.5618 $\mathrm{kW}$ y $0.4490 \mathrm{~kW}$, respectivamente. 
Figura 4 Solución \#2: códigos y calores residuales de los elementos combustibles de la MPC-32 número 24

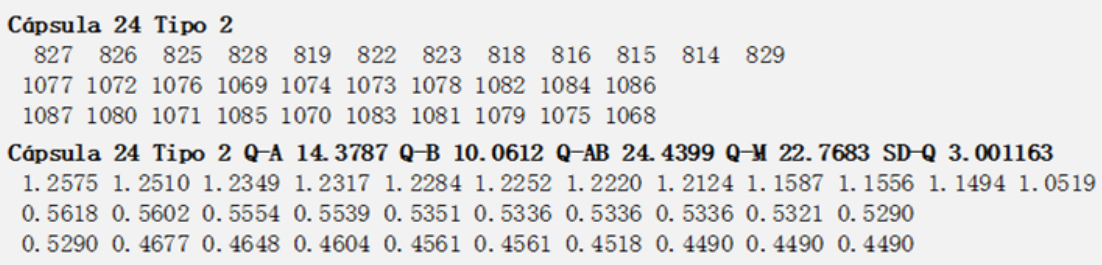

Otros resultados de interés mostrados en la Figura 4 son: (i) el calor residual en la MPC-32 número 24 que asciende a $24.4399 \mathrm{~kW}$, (ii) el calor residual en la región permisiva $\mathrm{R}_{\mathrm{a}}$ que es igual a $14.3787 \mathrm{~kW}$, (iii) el calor residual en la región severa $R_{b}$ cuyo valor es $10.0612 \mathrm{~kW}$, (iv) el calor residual medio, igual a $22.7683 \mathrm{~kW}$, correspondiente a la carga de elementos combustibles en los 37 contenedores que constituyen la solución \#2, y, finalmente, (v) la desviación tipo de la carga térmica asociada a la solución \#2, cuyo valor es $\sigma(\mathrm{Q})=3.001163$.

\section{Conclusiones y líneas de trabajo futuras}

La Programación lineal entera mixta se ha mostrado como una técnica de optimización competitiva para resolver problemas de minimización del coste de almacenaje de combustible nuclear. Esto se confirma a través de los experimentos que hemos realizado utilizando el modelo MILP-1 (diseñado para este trabajo e implementado en solucionador CPLEX) frente a la base de datos "ITEC-1394 Rev4.xlsm" suministrada por ENDESA. Dicha base permite generar instancias con dimensiones industriales (v.gr. 1200 elementos combustibles y de 1 a 30 tipos de contenedores regionalizados, lo cual se traduce en un número de tipos de regiones de calor de decaimiento comprendido entre 2 y 60 ).

El método propuesto aquí para el problema del encapsulamiento monoetápico del combustible nuclear gastado es computacionalmente competitivo en sus dos fases: programa matemático MILP-1 y algoritmo exacto A1. El modelo MILP-1 se utiliza para minimizar el coste de los contenedores requeridos para encapsular el combustible nuclear, ofreciendo como solución una asignación óptima de elementos combustibles a regiones térmicas virtuales, en menos de 0.5 segundos de CPU, usando instancias con 1500 elementos, 6 tipos de regiones y 4 tipos de contenedores. El algoritmo A1, que parte de una solución de MILP-1, es capaz de asignar los elementos a las regiones reales de los contenedores en menos de 0.25 segundos con los juegos de datos empleados.

En cuanto a investigaciones futuras, hemos previsto las siguientes líneas de actuación:

L1. Diseño de heurísticas relacionadas con el modelo MILP-1 para minimizar el coste de almacenamiento fijando el número de tipos de regiones térmicas
L2. Formulación y explotación de otros modelos basados en Programación lineal entera mixta para asignar directamente los elementos combustibles a las regiones térmicas de los contenedores según diversos criterios de optimización.

L3. Diseño de métodos heurísticos vinculados a la línea de trabajo L2 para equilibrar las cargas térmicas de los elementos combustibles en los contenedores [20].

L4. Extensión de los modelos y procedimientos de las líneas anteriores al caso de carga polietápica para un horizonte de explotación de la central nuclear.

\section{Agradecimientos:}

Este trabajo ha sido subvencionado por el Ministerio de Ciencia, Innovación y Universidades del Gobierno de España con el proyecto OPTHEUS (ref. PGC2018-095080B-I00), incluyendo Fondos para el desarrollo regional europeo, y el proyecto UPC "Desarrollo de herramientas para la simulación y optimización del vaciado de piscinas de combustible gastado de centrales nucleares" financiado por ENDESA Generación, S.A. Los autores agradecen también a la ASOCIACIÓN NUCLEAR ASCÓ-VANDELLÓS II (ANAV) el apoyo prestado y, especialmente, al Sr. Jordi Estrampes, jefe de Ingeniería del Reactor y Salvaguardias Nucleares de la CN Ascó, por sus consejos y aclaraciones.

\section{Referencias}

[1]Herranz LE, Penalva J, Feria F (2015) CFD analysis of a cask for spent fuel dry storage: Model fundamentals and sensitivity studies. Annals of Nuclear Energy, 76(2015): 54-62. https://doi.org/10.1016/j.anucene.2014.09.032.

[2] Wu Y, Klein J, Zhou H, Zuo L (2018) Thermal and fluid analysis of dry cask storage containers over multiple years of service. Annals of Nuclear Energy, 112(2018): 132-142. https://doi.org/10.1016/j.anucene.2017.10.013

[3] Sempértegui R, Bautista J, Griño R, Pereira J (2002) Models and procedures for Electric Energy Distribution Planning. A review. IFAC Proceedings Volumes, 35(1): 395400.

https://doi.org/10.3182/20020721-6-ES-1901.01217. 
[4] Paiva PC, Khodr HM, Dominguez-Navarro JA, Yusta JM, Urdaneta AJ (2005) Integral planning of primary-secondary distribution systems using mixed integer linear programming. IEEE Transactions on Power Systems 20 (2), 1134-1143.

https://doi.org/10.1109/TPWRS.2005.846108

[5] Georgilakis PS, Hatziargyriou ND (2015) A review of power distribution planning in the modern power systems era: Models, methods and future research. Electric Power Systems Research, 121(2015): 89-100.

https://doi.org/10.1016/j.epsr.2014.12.010.

[6] Klyapovskiy S, You S, Cai H, Bindner HW (2019) Incorporate flexibilit in distribution grid planning through a framework solution. International Journal of Electrical Power \& Energy Systems, 111(2019): 66-78. https://doi.org/10.1016/j.ijepes.2019.03.069.

[7] CSN (2020) El combustible nuclear y su ciclo. Monografía Consejo de Seguridad Nuclear.

https://www.csn.es/el-combustible-nuclear-y-su-ciclo.

[8] Merino AG, Gonzalez A (2019) Reliability methods in the design point of free-standing spent fuel racks under seismic conditions. Progress in nuclear energy 115, 208220. https://doi.org/10.1016/j.pnucene.2019.03.021

[9] Chen YS (2018). Thermal analysis for the integrated spent fuel pool of the Chinshan plant in the decommissioning process. Annals of Nuclear Energy 119, 163-174. https://doi.org/10.1016/j.anucene.2018.05.005

[10] Yoo HS, Yoo SH, Kim ES (2019) Heat transfer enhancement in dry cask storage for nuclear spent fuel using additive high density inert gas. Annals of Nuclear Energy 132, 108-118. https://doi.org/10.1016/j.anucene.2019.04.018

[11] Batet L, León P, Serra E, Ciruelos J, Estrampes J, Culebras F (2018) Desarrollo de una herramienta de ayuda a la gestión y optimización de carga de contenedores de combustible gastado. SNE 44 Reunión anual de la Sociedad Nuclear Española, 26-28 septiembre 2018, Ávila (Spain).

[12] CSN (2015) Propuesta de dictamen técnico para la aprobación de la revisión 2 del certificado del modelo de bulto de transporte HIStAR 100, solicitada por ENRESA. Informe CSN/TFCN/II/REV.2/E-0120.
[13] Ruiz López MC, Orozco Hernández G, Zamora Martín F, García Leiva M (2015) Contenedores para el almacenamiento temporal y el transporte del combustible gastado. aLFa (Revista de seguridad nuclear y protección radiológica. Consejo de Seguridad Nuclear) 27: 8-15. https://www.csn.es/documents/10182/13557/Alfa+27

[14] Penalva, JE; Feria, F; Herranz, LE (2014) Modelling of cladding thermal evolution along cask storage. Proceedings 22nd International Conference on Nuclear Engineering (ICONE22), 4, article Number: V004T08A012.

[15] Wu YJ, Klein J, Zhou HC, Zuo L (2018) Thermal and fl id analysis of dry cask storage containers over multiple years of service. Annals of Nuclear Energy 112, 132142. https://doi.org/10.1016/j.anucene.2017.10.013

[16] Bautista J, Batet L, Mateo M (2020) Minimización del coste de almacenaje de combustible nuclear gastado en cápsulas MPC-32 con programación lineal entera mixta: Caso de carga en una etapa. Technical report OPEWP.2020/01 (20200212). https://doi.org/10.13140/RG.2.2.36331.90407/1

[17] Bautista J (2020) Modelos y herramientas de decisión. Madrid: DEXTRA Editorial, (Capítulo 5).

[18] Holtec International (2008) FSAR, Final Safety Analysis Report for the HI-StORM 100 Cask System. Holtec Report No. HI-2002444, Rev. 7.

https:/www.nrc.gov/docs/ML0824/ML082401629.pdf

[17] Bautista J, Batet L, Mateo M (2020) Soluciones con programación lineal entera mixta para el almacenamiento monoetápico del combustible nuclear gastado en cápsulas MPC-32 en la central nuclear Ascó 1. Technical report OPE-WP.2020/02 (20200520). https://doi.org/10.13140/RG.2.2.14615.39845

[18] Spencer KY, Tsvetkov PV, Janell JJ (2019) Validation of the GAMMA-PC methodology for dry cask loading optimization. Progress in Nuclear Energy 117, 2019, 103038. https://doi.org/10.1016/j.pnucene.2019.04.009 
Anexo I: Instancia Ascó\#01_06

\begin{tabular}{|c|c|c|c|c|c|c|c|c|c|}
\hline$i$ & $q_{i}(T)$ & $i$ & $q_{i}(T)$ & $i$ & $q_{i}(T)$ & $i$ & $q_{i}(T)$ & $i$ & $q_{i}(T)$ \\
\hline 1 & 0.4189 & 41 & 0.4189 & 81 & 0.6098 & 121 & 0.6296 & 161 & 0.5789 \\
\hline 2 & 0.4189 & 42 & 0.4189 & 82 & 0.6098 & 122 & 0.6332 & 162 & 0.5302 \\
\hline 3 & 0.4189 & 43 & 0.4189 & 83 & 0.5789 & 123 & 0.6296 & 163 & 0.5858 \\
\hline 4 & 0.4189 & 44 & 0.4189 & 84 & 0.6010 & 124 & 0.5927 & 164 & 0.5335 \\
\hline 5 & 0.4189 & 45 & 0.4189 & 85 & 0.5772 & 125 & 0.5335 & 165 & 0.5351 \\
\hline 6 & 0.4189 & 46 & 0.4189 & 86 & 0.5236 & 126 & 0.6917 & 166 & 0.5327 \\
\hline 7 & 0.4189 & 47 & 0.4189 & 87 & 0.5302 & 127 & 0.6898 & 167 & 0.5434 \\
\hline 8 & 0.4189 & 48 & 0.4189 & 88 & 0.5302 & 128 & 0.6165 & 168 & 0.5418 \\
\hline 9 & 0.4189 & 49 & 0.4189 & 89 & 0.5772 & 129 & 0.6147 & 169 & 0.5302 \\
\hline 10 & 0.4189 & 50 & 0.4189 & 90 & 0.6049 & 130 & 0.7068 & 170 & 0.6955 \\
\hline 11 & 0.4189 & 51 & 0.4189 & 91 & 0.5962 & 131 & 0.4942 & 171 & 0.7052 \\
\hline 12 & 0.4189 & 52 & 0.4189 & 92 & 0.6101 & 132 & 0.6183 & 172 & 0.5055 \\
\hline 13 & 0.4189 & 53 & 0.4189 & 93 & 0.5302 & 133 & 0.6917 & 173 & 0.5039 \\
\hline 14 & 0.4189 & 54 & 0.5979 & 94 & 0.4772 & 134 & 0.4925 & 174 & 0.5813 \\
\hline 15 & 0.4189 & 55 & 0.5841 & 95 & 0.4820 & 135 & 0.6363 & 175 & 0.5088 \\
\hline 16 & 0.4189 & 56 & 0.5789 & 96 & 0.4756 & 136 & 0.4942 & 176 & 0.5270 \\
\hline 17 & 0.4189 & 57 & 0.5789 & 97 & 0.4836 & 137 & 0.4909 & 177 & 0.5844 \\
\hline 18 & 0.4189 & 58 & 0.5962 & 98 & 0.4740 & 138 & 0.4958 & 178 & 0.5064 \\
\hline 19 & 0.4189 & 59 & 0.5818 & 99 & 0.4836 & 139 & 0.4974 & 179 & 0.5253 \\
\hline 20 & 0.4189 & 60 & 0.6080 & 100 & 0.4756 & 140 & 0.7105 & 180 & 0.5031 \\
\hline 21 & 0.4189 & 61 & 0.5384 & 101 & 0.4756 & 141 & 0.4925 & 181 & 0.5072 \\
\hline 22 & 0.4189 & 62 & 0.6084 & 102 & 0.5335 & 142 & 0.7442 & 182 & 0.5270 \\
\hline 23 & 0.4189 & 63 & 0.5310 & 103 & 0.6350 & 143 & 0.6112 & 183 & 0.6917 \\
\hline 24 & 0.4189 & 64 & 0.5766 & 104 & 0.5979 & 144 & 0.6898 & 184 & 0.5831 \\
\hline 25 & 0.4189 & 65 & 0.5732 & 105 & 0.6296 & 145 & 0.4974 & 185 & 0.6973 \\
\hline 26 & 0.4189 & 66 & 0.5784 & 106 & 0.5962 & 146 & 0.5875 & 186 & 0.5023 \\
\hline 27 & 0.4189 & 67 & 0.6014 & 107 & 0.6385 & 147 & 0.5451 & 187 & 0.5253 \\
\hline 28 & 0.4189 & 68 & 0.5801 & 108 & 0.5997 & 148 & 0.5853 & 188 & 0.5848 \\
\hline 29 & 0.4189 & 69 & 0.5562 & 109 & 0.5962 & 149 & 0.5343 & 189 & 0.5990 \\
\hline 30 & 0.4189 & 70 & 0.5721 & 110 & 0.5979 & 150 & 0.5401 & 190 & 0.5467 \\
\hline 31 & 0.4189 & 71 & 0.5766 & 111 & 0.5335 & 151 & 0.5335 & 191 & 0.5973 \\
\hline 32 & 0.4189 & 72 & 0.5477 & 112 & 0.5997 & 152 & 0.5451 & 192 & 0.5533 \\
\hline 33 & 0.4189 & 73 & 0.5704 & 113 & 0.5979 & 153 & 0.5858 & 193 & 0.5973 \\
\hline 34 & 0.4189 & 74 & 0.5477 & 114 & 0.5962 & 154 & 0.5444 & 194 & 0.5500 \\
\hline 35 & 0.4189 & 75 & 0.5835 & 115 & 0.6860 & 155 & 0.5318 & 195 & 0.6571 \\
\hline 36 & 0.4189 & 76 & 0.5302 & 116 & 0.5351 & 156 & 0.5418 & 196 & 0.6571 \\
\hline 37 & 0.4189 & 77 & 0.5484 & 117 & 0.5962 & 157 & 0.5434 & 197 & 0.5417 \\
\hline 38 & 0.4189 & 78 & 0.5318 & 118 & 0.5940 & 158 & 0.5910 & 198 & 0.5417 \\
\hline 39 & 0.4189 & 79 & 0.5749 & 119 & 0.6347 & 159 & 0.5905 & 199 & 0.6535 \\
\hline 40 & 0.4189 & 80 & 0.6098 & 120 & 0.6010 & 160 & 0.5841 & 200 & 0.6094 \\
\hline
\end{tabular}

Tabla A1 (1 de 6): Conjunto de elementos combustibles correspondiente a la instancia ASCÓ\#01 6.0. Las características son: identificador i del elemento y calor residual $\mathrm{q}_{i}(\mathrm{~T})$ para $\mathrm{T}=6$ años. 


\begin{tabular}{|c|c|c|c|c|c|c|c|c|c|}
\hline$i$ & $q_{i}(T)$ & $i$ & $q_{i}(T)$ & $i$ & $q_{i}(T)$ & $l$ & $q_{i}(T)$ & $i$ & $q_{i}(T)$ \\
\hline 01 & 0.5533 & & 06160 & & 0.6320 & 0 & & 10 & $0<000$ \\
\hline 202 & 973 & 242 & 44 & 282 & 337 & 322 & 7203 & 362 & $69^{\circ}$ \\
\hline 03 & 6553 & 243 & 0.6442 & 283 & 6372 & 323 & 6904 & 363 & 83 \\
\hline 204 & 939 & 244 & 673 & 284 & 2 & 324 & 184 & 364 & 59 \\
\hline 205 & 5500 & 245 & 6028 & 285 & 512 & 325 & 7146 & 365 & 6915 \\
\hline 206 & 6025 & 246 & 93 & 286 & & 326 & 071 & 366 & 15 \\
\hline 207 & 5533 & 247 & 0.6285 & 287 & 99 & 327 & 0.7128 & 367 & 0.8274 \\
\hline 208 & 50 & 248 & & 288 & & 328 & & 368 & \\
\hline 209 & 6007 & 249 & 99 & 289 & 43 & 329 & 0.7109 & 369 & 315 \\
\hline 210 & 34 & 250 & 07 & 290 & & 330 & 90 & 370 & 212 \\
\hline 11 & 6172 & 251 & 977 & 291 & & 331 & 71 & 371 & 336 \\
\hline 212 & $\$ 1$ & 252 & & 292 & & 332 & & 372 & 233 \\
\hline 13 & 54 & 53 & & 293 & & 333 & & 373 & 482 \\
\hline 214 & 60 & 254 & 07 & 294 & & 334 & 22 & 374 & 784 \\
\hline 15 & 34 & 5 & & 295 & & 335 & & 375 & 710 \\
\hline 216 & 94 & 256 & 99 & 296 & & 336 & 46 & 376 & 759 \\
\hline 17 & & 7 & & 297 & & 3 & & 377 & \\
\hline 218 & 36 & 258 & 77 & 298 & & 338 & 57 & 378 & 735 \\
\hline 19 & 1 & 9 & & 299 & & & & 379 & \\
\hline 220 & 12 & 260 & 79 & 300 & & 340 & 75 & 380 & 759 \\
\hline 21 & 4 & 261 & & 301 & & 32 & & 381 & \\
\hline 222 & 119 & 262 & & 302 & & 342 & 57 & 382 & 239 \\
\hline 23 & 60 & 263 & & $30 ?$ & & 32 & & 383 & \\
\hline 224 & 154 & 264 & & 304 & & 344 & 75 & 384 & 258 \\
\hline 25 & 36 & 265 & 45 & 30 & & 32 & & 385 & \\
\hline 226 & 94 & 266 & & 306 & & 346 & & 386 & 57 \\
\hline 227 & 51 & 267 & 0. & 307 & & 32 & & 387 & \\
\hline 228 & $\$ 77$ & 268 & & 308 & & 348 & 61 & 388 & 599 \\
\hline 229 & 68 & 269 & 0 & 309 & & 349 & 61 & 389 & 38 \\
\hline 230 & 410 & 270 & & 310 & & 350 & 66 & 390 & 677 \\
\hline 231 & 351 & 271 & 20 & 311 & & 351 & 44 & 391 & 15 \\
\hline 232 & 0.6137 & 272 & 0.6 & 312 & & 352 & & 392 & 558 \\
\hline 233 & 0.6101 & 273 & 0. & 313 & & 353 & 520 & 393 & 638 \\
\hline 234 & 0.6442 & 274 & 0.6355 & 314 & & 354 & 0.6289 & 394 & 0.7638 \\
\hline 235 & 0.6303 & 275 & 0. & 315 & & 355 & 44 & 395 & 638 \\
\hline 236 & 0.6512 & 276 & 0.6372 & 316 & & 356 & 489 & 396 & 0.7284 \\
\hline 237 & 0.6165 & 277 & & 317 & & 357 & 07 & 397 & 0.7717 \\
\hline 238 & 0.6355 & 278 & 0.6 & 318 & & 358 & & 398 & 0.7638 \\
\hline 239 & & 279 & & 319 & & 359 & & 399 & 343 \\
\hline 240 & 0.6233 & 280 & 0.6372 & 320 & 0.7203 & 360 & 0.6839 & 400 & 0.7323 \\
\hline
\end{tabular}

Tabla A1 (2 de 6): Conjunto de elementos combustibles correspondiente a la instancia Ascó\#01_6.0. Las características son: identificador i del elemento y calor residual $\mathrm{q}_{\mathrm{i}}(\mathrm{T})$ para $\mathrm{T}=6$ años. 


\begin{tabular}{|c|c|c|c|c|c|c|c|c|c|}
\hline$i$ & $q_{i}(T)$ & $i$ & $q_{i}(T)$ & $i$ & $q_{i}(T)$ & $i$ & $q_{i}(T)$ & $i$ & $q_{i}(T)$ \\
\hline 401 & 0.8441 & 441 & 0.8820 & 481 & 0.7903 & 521 & 0.8030 & 561 & 0.8460 \\
\hline 402 & 0.7304 & 442 & 0.8952 & 482 & 0.8128 & 522 & 0.7968 & 562 & 1.0201 \\
\hline 403 & 0.7677 & 443 & 0.8864 & 483 & 0.7801 & 523 & 0.9134 & 563 & 0.7901 \\
\hline 404 & 0.7677 & 444 & 0.8886 & 484 & 0.8086 & 524 & 0.8974 & 564 & 1.0176 \\
\hline 405 & 0.7658 & 445 & 0.8908 & 485 & 0.8066 & 525 & 0.8134 & 565 & 1.0126 \\
\hline 406 & 0.7658 & 446 & 0.8842 & 486 & 0.8046 & 526 & 0.8303 & 566 & 0.7989 \\
\hline 407 & 0.7483 & 447 & 0.8908 & 487 & 0.8256 & 527 & 0.8345 & 567 & 1.0226 \\
\hline 408 & 0.7386 & 448 & 0.8886 & 488 & 0.8128 & 528 & 0.8367 & 568 & 0.7814 \\
\hline 409 & 0.7638 & 449 & 0.9063 & 489 & 0.8107 & 529 & 0.8345 & 569 & 0.7836 \\
\hline 410 & 0.7463 & 450 & 0.7704 & 490 & 0.7882 & 530 & 0.8303 & 570 & 0.7923 \\
\hline 411 & 0.7483 & 451 & 0.7644 & 491 & 0.8213 & 531 & 0.8113 & 571 & 0.7879 \\
\hline 412 & 0.7483 & 452 & 0.7724 & 492 & 0.8046 & 532 & 0.8134 & 572 & 1.0126 \\
\hline 413 & 0.7521 & 453 & 0.7704 & 493 & 0.7903 & 533 & 0.8197 & 573 & 1.0151 \\
\hline 414 & 0.7502 & 454 & 0.7882 & 494 & 0.8149 & 534 & 0.8093 & 574 & 1.0076 \\
\hline 415 & 0.7560 & 455 & 0.7700 & 495 & 0.8087 & 535 & 0.8155 & 575 & 0.7901 \\
\hline 416 & 0.7463 & 456 & 0.7720 & 496 & 0.8192 & 536 & 0.8218 & 576 & 0.7749 \\
\hline 417 & 0.7463 & 457 & 0.7781 & 497 & 0.7882 & 537 & 0.8218 & 577 & 1.0201 \\
\hline 418 & 0.7502 & 458 & 0.7744 & 498 & 1.0027 & 538 & 0.8303 & 578 & 1.0076 \\
\hline 419 & 0.7502 & 459 & 0.7781 & 499 & 0.9203 & 539 & 0.8093 & 579 & 1.0026 \\
\hline 420 & 0.7463 & 460 & 0.7680 & 500 & 0.9955 & 540 & 0.8155 & 580 & 1.0026 \\
\hline 421 & 0.7406 & 461 & 0.7943 & 501 & 0.9955 & 541 & 0.8239 & 581 & 1.0026 \\
\hline 422 & 0.7776 & 462 & 0.7923 & 502 & 0.9249 & 542 & 0.8261 & 582 & 0.8483 \\
\hline 423 & 0.7736 & 463 & 0.7882 & 503 & 1.0027 & 543 & 0.8197 & 583 & 0.8329 \\
\hline 424 & 0.7795 & 464 & 0.7842 & 504 & 0.9180 & 544 & 0.8134 & 584 & 0.8329 \\
\hline 425 & 0.7677 & 465 & 0.7903 & 505 & 0.9111 & 545 & 0.8218 & 585 & 0.8417 \\
\hline 426 & 0.7680 & 466 & 0.7842 & 506 & 0.9180 & 546 & 0.8439 & 586 & 0.8395 \\
\hline 427 & 0.7720 & 467 & 0.7903 & 507 & 0.9295 & 547 & 0.8218 & 587 & 0.8505 \\
\hline 428 & 0.7620 & 468 & 0.7882 & 508 & 0.9931 & 548 & 0.8527 & 588 & 0.8329 \\
\hline 429 & 0.7560 & 469 & 0.7903 & 509 & 0.9907 & 549 & 0.8351 & 589 & 0.8549 \\
\hline 430 & 0.7640 & 470 & 0.8579 & 510 & 0.9835 & 550 & 0.8197 & 590 & 0.8615 \\
\hline 431 & 0.7540 & 471 & 0.7680 & 511 & 0.9157 & 551 & 0.8395 & 591 & 0.8483 \\
\hline 432 & 0.7640 & 472 & 0.7720 & 512 & 0.9157 & 552 & 0.8197 & 592 & 0.8527 \\
\hline 433 & 0.7640 & 473 & 0.8601 & 513 & 0.9835 & 553 & 0.8155 & 593 & 0.8571 \\
\hline 434 & 0.8666 & 474 & 0.8514 & 514 & 1.1306 & 554 & 0.8218 & 594 & 0.8460 \\
\hline 435 & 0.8558 & 475 & 0.7700 & 515 & 1.1223 & 555 & 0.8218 & 595 & 0.8505 \\
\hline 436 & 0.8623 & 476 & 0.7761 & 516 & 1.1278 & 556 & 0.8483 & 596 & 0.8571 \\
\hline 437 & 0.8623 & 477 & 0.8492 & 517 & 1.1389 & 557 & 0.8460 & 597 & 0.8571 \\
\hline 438 & 0.8558 & 478 & 0.7903 & 518 & 0.8030 & 558 & 0.8373 & 598 & 0.8929 \\
\hline 439 & 0.8710 & 479 & 0.7923 & 519 & 0.8997 & 559 & 0.8197 & 599 & 0.8816 \\
\hline 440 & 0.8688 & 480 & 0.7943 & 520 & 0.8907 & 560 & 0.8218 & 600 & 0.8839 \\
\hline
\end{tabular}

Tabla A1 (3 de 6): Conjunto de elementos combustibles correspondiente a la instancia Ascó\#01_6.0. Las características son: identificador i del elemento y calor residual $q_{i}(T)$ para $T=6$ años. 


\begin{tabular}{|c|c|c|c|c|c|c|c|c|c|}
\hline$l$ & $q_{i}(T)$ & $i$ & $q_{i}(T)$ & $l$ & $q_{i}(T)$ & l & $q_{i}(T)$ & $l$ & $q_{i}(T)$ \\
\hline 601 & 0.8839 & 41 & 1.0679 & 81 & 0.9130 & 721 & 09762 & 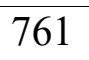 & \\
\hline 602 & 285 & 2 & & & & 722 & & 62 & \\
\hline 13 & 3929 & 43 & 412 & 683 & 036 & 723 & 660 & 763 & 368 \\
\hline 604 & 861 & 44 & 254 & 84 & 177 & 724 & 62 & 764 & 9667 \\
\hline 95 & 3460 & 45 & 0733 & 85 & 130 & 725 & 716 & 765 & 0631 \\
\hline 606 & 51 & 46 & 96 & 686 & 59 & 726 & & 766 & 547 \\
\hline 607 & 3351 & 647 & 78 & 87 & 201 & 727 & 59 & 767 & .0491 \\
\hline 608 & 39 & 48 & 68 & 688 & 12 & 728 & & 768 & 575 \\
\hline 09 & 8907 & 49 & 78 & 689 & 89 & 729 & & 769 & 830 \\
\hline 10 & 71 & 650 & 65 & 690 & 10 & 730 & & 770 & 1.0688 \\
\hline 1 & 49 & 1 & 72 & 691 & & 1 & & 771 & 60 \\
\hline 612 & 27 & 652 & 95 & 692 & 012 & 732 & & 772 & 45 \\
\hline & & 3 & & 3 & & 3 & & 3 & \\
\hline 14 & 16 & 54 & 95 & 694 & 14 & 734 & & 774 & 45 \\
\hline & & & & 5 & & & & 5 & \\
\hline 16 & 49 & 656 & 65 & 696 & 36 & 736 & 75 & 776 & 1.0716 \\
\hline & & & & 7 & & & & 7 & \\
\hline 18 & 15 & 658 & 36 & 698 & 67 & 738 & & 778 & 543 \\
\hline & & & & 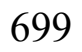 & & & & 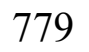 & \\
\hline 20 & 49 & 660 & 18 & 700 & 96 & 740 & & 780 & 1.0773 \\
\hline 1 & & 1 & & 1 & & & & 1 & \\
\hline 22 & 04 & 662 & 43 & 702 & 28 & 742 & & 782 & 1.2575 \\
\hline 3 & & & & 03 & 57 & & & 3 & \\
\hline 24 & 90 & 6 & 79 & 704 & 06 & 744 & & 784 & 45 \\
\hline 5 & 49 & 6 & & 05 & 06 & 745 & & 785 & 73 \\
\hline 6 & 6 & & 06 & 70 & & 7 & & 786 & 87 \\
\hline 27 & 58 & 667 & 81 & 707 & 85 & 747 & & 787 & 73 \\
\hline 6 & 66 & & 83 & 70 & 67 & 74 & & 788 & 1.0944 \\
\hline 29 & 41 & 669 & 99 & 09 & 33 & 749 & & 789 & 543 \\
\hline & 68 & & 60 & 71 & & 750 & & 790 & 1.0916 \\
\hline 631 & 39 & 671 & 83 & 11 & 83 & 751 & 40 & 791 & 1.0916 \\
\hline & 14 & & 06 & 71 & 08 & 752 & 38 & 792 & 1.2608 \\
\hline 33 & 0652 & 673 & 72 & 713 & 84 & 753 & 34 & 793 & 1.2608 \\
\hline & 19 & & 52 & 71 & 33 & 754 & 75 & 794 & 1.2608 \\
\hline 635 & 1.0 & 675 & 65 & 71 & 75 & 755 & 35 & 795 & 1.0944 \\
\hline 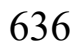 & 281 & 67 & 43 & 716 & 34 & 756 & 36 & 796 & 1.0887 \\
\hline 637 & 1.0412 & 677 & 0.9059 & 717 & 0.9458 & 757 & 1.3301 & 797 & 0.8749 \\
\hline 62 & 39 & & 36 & $71 \varepsilon$ & 46 & 758 & 1.3503 & 798 & 1.1093 \\
\hline 639 & 1.0333 & 679 & 0.9012 & 719 & 1.1204 & 759 & 1.3672 & 799 & 0.8724 \\
\hline 640 & 1.0733 & 680 & 0.9059 & 720 & 0.9508 & 760 & 0.9721 & 800 & 0.8698 \\
\hline
\end{tabular}

Tabla A1 (4 de 6): Conjunto de elementos combustibles correspondiente a la instancia Ascó\#01_6.0. Las características son: identificador i del elemento y calor residual $\mathrm{q}_{\mathrm{i}}(\mathrm{T})$ para $\mathrm{T}=6$ años. 


\begin{tabular}{|c|c|c|c|c|c|c|c|c|c|}
\hline$i$ & $q_{i}(T)$ & $i$ & $q_{i}(T)$ & $i$ & $q_{i}(T)$ & $i$ & $q_{i}(T)$ & $i$ & $q_{i}(T)$ \\
\hline 801 & 1.1093 & 841 & 1.2124 & 881 & 0.4651 & 921 & 0.4902 & 961 & 0.5179 \\
\hline 802 & 0.8826 & 842 & 1.2478 & 882 & 0.4603 & 922 & 0.4934 & 962 & 0.4312 \\
\hline 803 & 1.1124 & 843 & 1.2060 & 883 & 0.5223 & 923 & 0.5558 & 963 & 0.5148 \\
\hline 804 & 1.1185 & 844 & 1.1997 & 884 & 0.4571 & 924 & 0.5507 & 964 & 0.5179 \\
\hline 805 & 1.1154 & 845 & 1.2156 & 885 & 0.4651 & 925 & 0.4885 & 965 & 0.4439 \\
\hline 806 & 1.1185 & 846 & 1.2029 & 886 & 0.5156 & 926 & 0.5167 & 966 & 0.4397 \\
\hline 807 & 0.8673 & 847 & 1.2124 & 887 & 0.5426 & 927 & 0.4546 & 967 & 0.4213 \\
\hline 808 & 0.8749 & 848 & 1.2446 & 888 & 0.5273 & 928 & 0.4771 & 968 & 0.5256 \\
\hline 809 & 0.8673 & 849 & 1.2510 & 889 & 0.5240 & 929 & 0.4755 & 969 & 0.4368 \\
\hline 810 & 1.1124 & 850 & 1.2060 & 890 & 0.4492 & 930 & 0.5576 & 970 & 0.4326 \\
\hline 811 & 1.1093 & 851 & 1.2092 & 891 & 0.4196 & 931 & 0.5167 & 971 & 0.4656 \\
\hline 812 & 0.8749 & 852 & 1.2381 & 892 & 0.5206 & 932 & 0.4117 & 972 & 0.5148 \\
\hline 813 & 1.1556 & 853 & 0.5923 & 893 & 0.4555 & 933 & 0.4656 & 973 & 0.4641 \\
\hline 814 & 1.1494 & 854 & 0.5947 & 894 & 0.5223 & 934 & 0.5015 & 974 & 0.5133 \\
\hline 815 & 1.1556 & 855 & 0.5947 & 895 & 0.4667 & 935 & 0.4312 & 975 & 0.5027 \\
\hline 816 & 1.1587 & 856 & 0.5923 & 896 & 0.4603 & 936 & 0.6366 & 976 & 0.5012 \\
\hline 817 & 0.5207 & 857 & 0.4069 & 897 & 0.4571 & 937 & 0.6114 & 977 & 0.4354 \\
\hline 818 & 1.2124 & 858 & 0.3913 & 898 & 0.4135 & 938 & 0.6120 & 978 & 0.4583 \\
\hline 819 & 1.2284 & 859 & 0.5159 & 899 & 0.5477 & 939 & 0.4651 & 979 & 0.4354 \\
\hline 820 & 0.5186 & 860 & 0.3975 & 900 & 0.5140 & 940 & 0.5190 & 980 & 0.4598 \\
\hline 821 & 0.5123 & 861 & 0.4292 & 901 & 0.5409 & 941 & 0.4731 & 981 & 0.5240 \\
\hline 822 & 1.2252 & 862 & 0.3975 & 902 & 0.4196 & 942 & 0.5257 & 982 & 0.4368 \\
\hline 823 & 1.2220 & 863 & 0.3975 & 903 & 0.5173 & 943 & 0.4635 & 983 & 0.4326 \\
\hline 824 & 0.5207 & 864 & 0.3975 & 904 & 0.5460 & 944 & 0.4476 & 984 & 0.4997 \\
\hline 825 & 1.2349 & 865 & 0.4038 & 905 & 0.5206 & 945 & 0.5477 & 985 & 0.5256 \\
\hline 826 & 1.2510 & 866 & 0.4785 & 906 & 0.4619 & 946 & 0.4683 & 986 & 0.5287 \\
\hline 827 & 1.2575 & 867 & 0.4022 & 907 & 0.5140 & 947 & 0.5210 & 987 & 0.5012 \\
\hline 828 & 1.2317 & 868 & 0.4651 & 908 & 0.5190 & 948 & 0.5012 & 988 & 0.4997 \\
\hline 829 & 1.0519 & 869 & 0.3975 & 909 & 0.4539 & 949 & 0.4354 & 989 & 0.5318 \\
\hline 830 & 1.2349 & 870 & 0.5133 & 910 & 0.4587 & 950 & 0.4700 & 990 & 0.5302 \\
\hline 831 & 1.2641 & 871 & 0.5234 & 911 & 0.4603 & 951 & 0.5012 & 991 & 0.5042 \\
\hline 832 & 1.0609 & 872 & 0.4372 & 912 & 0.4089 & 952 & 0.4656 & 992 & 0.5019 \\
\hline 833 & 1.0639 & 873 & 0.4836 & 913 & 0.5240 & 953 & 0.4744 & 993 & 0.5925 \\
\hline 834 & 1.0639 & 874 & 0.4325 & 914 & 0.5140 & 954 & 0.4554 & 994 & 0.5033 \\
\hline 835 & 1.2575 & 875 & 0.5477 & 915 & 0.4594 & 955 & 0.4298 & 995 & 0.4945 \\
\hline 836 & 1.2349 & 876 & 0.4635 & 916 & 0.4820 & 956 & 0.4554 & 996 & 0.5957 \\
\hline 837 & 1.2543 & 877 & 0.5223 & 917 & 0.4820 & 957 & 0.4656 & 997 & 0.4931 \\
\hline 838 & 1.2092 & 878 & 0.4524 & 918 & 0.4787 & 958 & 0.4729 & 998 & 0.5019 \\
\hline 839 & 1.2478 & 879 & 0.5426 & 919 & 0.4562 & 959 & 0.4368 & 999 & 0.4901 \\
\hline 840 & 0.5375 & 880 & 0.5375 & 920 & 0.5455 & 960 & 0.4714 & 1000 & 0.4887 \\
\hline
\end{tabular}

Tabla A1 (5 de 6): Conjunto de elementos combustibles correspondiente a la instancia Ascó\#01_6.0. Las características son: identificador i del elemento y calor residual $\mathrm{q}_{\mathrm{i}}(\mathrm{T})$ para $\mathrm{T}=6$ años. 


\begin{tabular}{|c|c|c|c|c|c|c|c|}
\hline$i$ & $q_{i}(T)$ & $i$ & $q_{i}(T)$ & $i$ & $q_{i}(T)$ & $i$ & $q_{i}(T)$ \\
\hline 1001 & 0.4960 & 1042 & 0.5713 & 1083 & 0.4561 & 1124 & 0.6924 \\
\hline 1002 & 0.5033 & 1043 & 0.4706 & 1084 & 0.5321 & 1125 & 0.6690 \\
\hline 1003 & 0.4901 & 1044 & 0.4691 & 1085 & 0.4604 & 1126 & 0.6654 \\
\hline 1004 & 0.5048 & 1045 & 0.4576 & 1086 & 0.5290 & 1127 & 0.6672 \\
\hline 1005 & 0.4989 & 1046 & 0.5681 & 1087 & 0.5290 & 1128 & 0.6690 \\
\hline 1006 & 0.6005 & 1047 & 0.4547 & 1088 & 0.4764 & 1129 & 0.67 \\
\hline 1007 & 0.4960 & 1048 & 0.4590 & 1089 & 0.4750 & 1130 & 0.6672 \\
\hline 1008 & 0.6021 & 1049 & 0.5713 & 1090 & 0.5649 & 1131 & 0.6690 \\
\hline 1009 & 0.5989 & 1050 & 0.4706 & 1091 & 0.5539 & 1132 & 0.6708 \\
\hline 010 & 0.4887 & 1051 & 0.4750 & 1092 & 0.5398 & 1133 & 0.7097 \\
\hline 1011 & 0.5989 & 1052 & 0.4735 & 1093 & 0.5554 & 1134 & 0.6407 \\
\hline 1012 & 0.4975 & 1053 & 0.4720 & 1094 & 0.4604 & 1135 & 0.71 \\
\hline 1013 & 0.5973 & 1054 & 0.4532 & 1095 & 0.4706 & 1136 & 0.7184 \\
\hline 1014 & 0.6053 & 1055 & 0.4794 & 1096 & 0.4720 & 1137 & 0.7222 \\
\hline 1015 & 0.5470 & 1056 & 0.4750 & 1097 & 0.4547 & 1138 & 0.7146 \\
\hline 16 & 0.5078 & 1057 & 0.5382 & 1098 & 0.5602 & 1139 & 0.7203 \\
\hline 1017 & 0.5122 & 1058 & 0.5305 & 1099 & 0.4677 & 1140 & 0.6794 \\
\hline 1018 & 0.5063 & 1059 & 0.4662 & 1100 & 0.4461 & 1141 & 0.6739 \\
\hline 1019 & 0.5348 & 1060 & 0.4504 & 1101 & 0.4677 & 1142 & 0.6720 \\
\hline 1020 & 0.5424 & 1061 & 0.4518 & 1102 & 0.4518 & 1143 & 0.7146 \\
\hline 21 & & & & 11 & & 112 & \\
\hline 022 & 0.4613 & 1063 & 0.5382 & 1104 & 0.4677 & 1145 & 0.7203 \\
\hline 1023 & 0.4628 & 1064 & 0.4576 & 1105 & 0.6512 & 1146 & 0.7165 \\
\hline 1024 & 0.4585 & 1065 & 0.4633 & 1106 & 0.6460 & 1147 & 0.7165 \\
\hline 1025 & 0.5394 & 1066 & 0.4619 & 1107 & 0.6407 & 1148 & 0.6739 \\
\hline 1026 & 0.4628 & 1067 & 0.4619 & 1108 & 0.6372 & 1149 & 0.6720 \\
\hline 27 & 0.4599 & 1068 & 0.4490 & 1109 & 0.6407 & 1150 & 0.7053 \\
\hline 028 & 0.5078 & 1069 & 0.5539 & 1110 & 0.6390 & 1151 & 0.7260 \\
\hline 1029 & 0.5152 & 1070 & 0.4561 & 1111 & 0.6477 & 1152 & 0.6684 \\
\hline 1030 & 0.5078 & 1071 & 0.4648 & 1112 & 0.6390 & 1153 & 0.7203 \\
\hline 1031 & 0.5122 & 1072 & 0.5602 & 1113 & 0.6337 & 1154 & 0.7184 \\
\hline 32 & 0.4599 & 1073 & 0.5336 & 1114 & 0.6530 & 1155 & 0.6734 \\
\hline 033 & 0.4599 & 1074 & 0.5351 & 1115 & 0.6565 & 1156 & 0.7146 \\
\hline 034 & 0.5409 & 1075 & 0.4490 & 1116 & 0.6601 & 1157 & 0.7090 \\
\hline 1035 & 0.4585 & 1076 & 0.5554 & 1117 & 0.6548 & 1158 & 0.7260 \\
\hline 1036 & 0.5152 & 1077 & 0.5618 & 1118 & 0.6495 & 1159 & 0.7593 \\
\hline 1037 & 0.5363 & 1078 & 0.5336 & 1119 & 0.6548 & 1160 & 0.7494 \\
\hline 38 & 5048 & 1079 & 4490 & 1120 & 0.6601 & 1161 & 0.7475 \\
\hline 039 & 0.5409 & 1080 & 0.4677 & 1121 & 0.6905 & 1162 & 0.7279 \\
\hline 340 & 0.5237 & 1081 & 0.4518 & 1122 & 0.6924 & 1163 & 0.7534 \\
\hline 1041 & 0.5713 & 1082 & 0.5336 & 1123 & 0.6924 & 1164 & 0.7713 \\
\hline
\end{tabular}

Tabla A1 (6 de 6): Conjunto de elementos combustibles correspondiente a la instancia Ascó\#01_6.0. Las características son: identificador i del elemento y calor residual $\mathrm{q}_{\mathrm{i}}(\mathrm{T})$ para $\mathrm{T}=6$ años. 


\section{Anexo II: Configuración de cápsulas Solución \#2}

Solución \#2 (3 1 2): Listado de carga de elementos combustibles en cápsulas MPC-32

Cápsula 1 Tipo 1

$\begin{array}{rrrrrrrrrrrr}751 & 761 & 759 & 752 & 758 & 756 & 762 & 750 & 763 & 755 & 757 & 753 \\ 41 & 42 & 45 & 47 & 48 & 49 & 52 & 53 & 9 & 18 & & \\ 27 & 28 & 29 & 30 & 31 & 33 & 34 & 35 & 36 & 37 & & \end{array}$

Cápsula 2 Tipo 1

$\begin{array}{llllllllllll}831 & 849 & 842 & 839 & 848 & 841 & 847 & 851 & 850 & 843 & 846 & 844\end{array}$

$\begin{array}{llllllllll}1051 & 1056 & 896 & 1033 & 1024 & 1035 & 897 & 884 & 919 & 878\end{array}$

$\begin{array}{llllllllll}1061 & 1060 & 982 & 977 & 967 & 902 & 891 & 932 & 912 & 860\end{array}$

Cápsula 3 Tipo 2

$\begin{array}{rrrrrrrrrrrr}255 & 244 & 285 & 259 & 263 & 242 & 250 & 254 & 278 & 239 & 269 & 272 \\ 14 & 15 & 16 & 17 & 19 & 20 & 21 & 22 & 1 & 2 & & \\ 3 & 4 & 5 & 6 & 7 & 8 & 10 & 11 & 12 & 13 & & \end{array}$

Cápsula 4 Tipo 2

$\begin{array}{rrrrrrrrrrrr}307 & 318 & 313 & 310 & 287 & 311 & 321 & 306 & 301 & 294 & 291 & 299 \\ 60 & 54 & 58 & 55 & 59 & 57 & 56 & 39 & 40 & 43 & & \\ 44 & 46 & 50 & 51 & 23 & 24 & 25 & 26 & 32 & 38 & & \end{array}$

Cápsula 5 Tipo 2

$\begin{array}{llllllllllll}366 & 325 & 327 & 329 & 330 & 331 & 333 & 323 & 361 & 341 & 358 & 338\end{array}$

$\begin{array}{llllllllll}80 & 62 & 67 & 75 & 68 & 66 & 64 & 71 & 79 & 65\end{array}$

$\begin{array}{llllllllll}70 & 73 & 69 & 77 & 72 & 74 & 61 & 78 & 63 & 76\end{array}$

Cápsula 6 Tipo 2

$\begin{array}{rrrrrrrrrrrr}381 & 377 & 379 & 374 & 380 & 376 & 378 & 375 & 368 & 372 & 370 & 383 \\ 92 & 82 & 81 & 90 & 84 & 91 & 83 & 89 & 85 & 87 & & \\ 88 & 93 & 86 & 97 & 99 & 95 & 94 & 96 & 100 & 98 & & \end{array}$

Cápsula 7 Tipo 2

$\begin{array}{llllllllllll}403 & 392 & 406 & 398 & 389 & 394 & 409 & 411 & 412 & 416 & 400 & 385 \\ 105 & 121 & 123 & 120 & 112 & 108 & 110 & 104 & 113 & 117 & & \\ 109 & 106 & 114 & 118 & 124 & 116 & 102 & 111 & 125 & 101 & & \end{array}$

Cápsula 8 Tipo 2

$\begin{array}{llllllllllll}449 & 445 & 444 & 441 & 440 & 434 & 422 & 426 & 428 & 419 & 418 & 420\end{array}$

$\begin{array}{llllllllll}132 & 128 & 129 & 143 & 146 & 153 & 148 & 152 & 147 & 150\end{array}$

$\begin{array}{llllllllll}149 & 151 & 139 & 145 & 138 & 131 & 136 & 134 & 141 & 137\end{array}$

Cápsula 9 Tipo 2

$\begin{array}{llllllllllll}474 & 489 & 485 & 486 & 462 & 468 & 457 & 452 & 453 & 450 & 460 & 451 \\ 158 & 159 & 163 & 160 & 174 & 161 & 154 & 167 & 157 & 168 & & \\ 156 & 165 & 164 & 166 & 155 & 169 & 162 & 175 & 172 & 173 & & \end{array}$

Cápsula 10 Tipo 2

$\begin{array}{llllllllllll}517 & 514 & 516 & 515 & 498 & 503 & 501 & 500 & 508 & 509 & 510 & 513 \\ 200 & 189 & 193 & 191 & 188 & 177 & 184 & 192 & 194 & 190 & & \\ 198 & 197 & 182 & 176 & 179 & 187 & 181 & 178 & 180 & 186 & & \end{array}$

Cápsula 11 Tipo 2

$\begin{array}{llllllllllll}567 & 562 & 577 & 564 & 573 & 565 & 572 & 574 & 578 & 579 & 580 & 581\end{array}$

$\begin{array}{llllllllll}211 & 213 & 215 & 222 & 219 & 206 & 209 & 202 & 204 & 208\end{array}$

$\begin{array}{llllllllll}207 & 201 & 205 & 221 & 217 & 216 & 210 & 214 & 212 & 218\end{array}$

Cápsula 12 Tipo 2

$\begin{array}{llllllllllll}628 & 626 & 627 & 629 & 630 & 632 & 633 & 631 & 623 & 614 & 619 & 624 \\ 235 & 247 & 240 & 252 & 249 & 237 & 224 & 232 & 233 & 248 & & \\ 245 & 251 & 226 & 228 & 230 & 229 & 223 & 231 & 227 & 225 & & \end{array}$




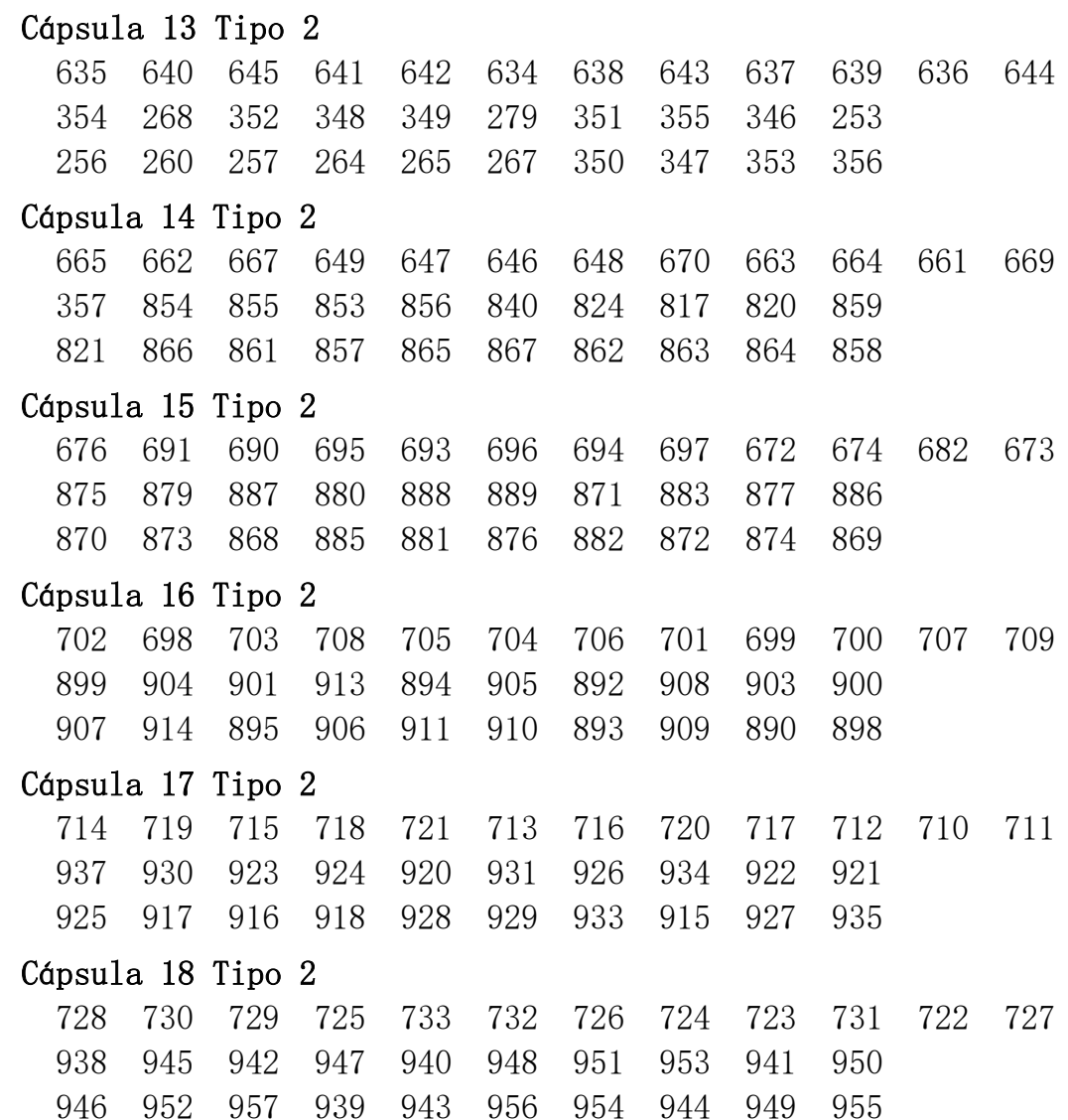

Cápsula 19 Tipo 2

$\begin{array}{llllllllllll}744 & 735 & 736 & 743 & 738 & 745 & 734 & 742 & 739 & 737 & 740 & 741\end{array}$ $\begin{array}{llllllllll}968 & 964 & 961 & 963 & 972 & 974 & 975 & 976 & 958 & 960\end{array}$ $\begin{array}{llllllllll}971 & 973 & 978 & 965 & 966 & 969 & 959 & 979 & 970 & 962\end{array}$

Cápsula 20 Tipo 2

$\begin{array}{llllllllllll}769 & 765 & 748 & 768 & 766 & 767 & 746 & 747 & 754 & 760 & 764 & 749\end{array}$ $\begin{array}{llllllllll}996 & 993 & 989 & 990 & 986 & 985 & 981 & 991 & 994 & 992\end{array}$ $\begin{array}{llllllllll}998 & 987 & 984 & 988 & 995 & 997 & 999 & 1000 & 980 & 983\end{array}$

\section{Cápsula 21 Tipo 2}

$\begin{array}{llllllllllll}778 & 779 & 777 & 780 & 772 & 774 & 773 & 776 & 770 & 781 & 771 & 775\end{array}$

$\begin{array}{llllllllll}1014 & 1008 & 1006 & 1011 & 1009 & 1013 & 1015 & 1020 & 1019 & 1017\end{array}$

1016101810041002100510121001100710031010

Cápsula 22 Tipo 2

$\begin{array}{llllllllllll}792 & 793 & 782 & 789 & 783 & 787 & 785 & 788 & 790 & 791 & 786 & 784\end{array}$

$\begin{array}{llllllllll}1041 & 1042 & 1034 & 1039 & 1021 & 1025 & 1037 & 1040 & 1036 & 1029\end{array}$

$\begin{array}{llllllllll}1031 & 1028 & 1030 & 1038 & 1043 & 1026 & 1023 & 1022 & 1032 & 1027\end{array}$

Cápsula 23 Tipo 2

$\begin{array}{llllllllllll}794 & 813 & 806 & 804 & 805 & 810 & 803 & 798 & 811 & 801 & 795 & 796\end{array}$

$\begin{array}{llllllllll}1049 & 1046 & 1057 & 1063 & 1058 & 1055 & 1052 & 1053 & 1050 & 1044\end{array}$

$10591065106610671048106410451047 \quad 10541062$

\section{Cápsula 24 Tipo 2}

$\begin{array}{llllllllllll}827 & 826 & 825 & 828 & 819 & 822 & 823 & 818 & 816 & 815 & 814 & 829\end{array}$

$\begin{array}{llllllllll}1077 & 1072 & 1076 & 1069 & 1074 & 1073 & 1078 & 1082 & 1084 & 1086\end{array}$

$\begin{array}{llllllllll}1087 & 1080 & 1071 & 1085 & 1070 & 1083 & 1081 & 1079 & 1075 & 1068\end{array}$

Cápsula 25 Tipo 2

$\begin{array}{llllllllllll}835 & 837 & 852 & 830 & 836 & 845 & 838 & 833 & 834 & 832 & 1090 & 1098\end{array}$

$1093109110921103108810891096 \quad 109511011099$

$11041094109711021100 \quad 0 \quad 000000$ 
Cápsula 26 Tipo 3

$\begin{array}{llllllllllll}246 & 142 & 220 & 140 & 130 & 171 & 185 & 170 & 126 & 133 & 183 & 127\end{array}$

$\begin{array}{lllllllllll}144 & 115 & 195 & 196 & 203 & 199 & 236 & 258 & 241 & 234\end{array}$

$\begin{array}{llllllllll}243 & 261 & 266 & 262 & 107 & 135 & 238 & 103 & 119 & 122\end{array}$

Cápsula 27 Tipo 3

$\begin{array}{llllllllllll}304 & 303 & 308 & 290 & 315 & 288 & 312 & 305 & 309 & 314 & 300 & 289\end{array}$

$\begin{array}{llllllllll}293 & 302 & 297 & 296 & 292 & 295 & 286 & 298 & 284 & 276\end{array}$

$\begin{array}{llllllllll}280 & 283 & 277 & 274 & 275 & 282 & 281 & 273 & 270 & 271\end{array}$

\section{Cápsula 28 Tipo 3}

$\begin{array}{llllllllllll}373 & 386 & 371 & 369 & 367 & 384 & 382 & 334 & 320 & 322 & 324 & 335 \\ 319 & 336 & 337 & 332 & 326 & 328 & 364 & 362 & 316 & 365 & & \\ 359 & 317 & 363 & 360 & 339 & 343 & 344 & 340 & 345 & 342 & & \end{array}$

Cápsula 29 Tipo 3

$\begin{array}{llllllllllll}436 & 435 & 401 & 387 & 391 & 424 & 423 & 427 & 397 & 390 & 404 & 425 \\ 405 & 433 & 430 & 432 & 395 & 393 & 388 & 429 & 415 & 431 & & \\ 413 & 414 & 407 & 417 & 410 & 421 & 408 & 399 & 402 & 396 & & \end{array}$

Cápsula 30 Tipo 3

$\begin{array}{llllllllllll}442 & 447 & 448 & 443 & 446 & 439 & 437 & 473 & 470 & 438 & 477 & 482\end{array}$

$\begin{array}{llllllllll}480 & 461 & 479 & 478 & 465 & 467 & 481 & 469 & 463 & 454\end{array}$

$\begin{array}{llllllllll}464 & 466 & 459 & 476 & 458 & 456 & 472 & 475 & 455 & 471\end{array}$

Cápsula 31 Tipo 3

$\begin{array}{llllllllllll}507 & 502 & 499 & 506 & 504 & 511 & 512 & 523 & 505 & 519 & 524 & 520 \\ 528 & 527 & 529 & 526 & 487 & 491 & 496 & 494 & 525 & 488 & & \\ 495 & 484 & 492 & 521 & 518 & 522 & 493 & 490 & 497 & 483 & & \end{array}$

Cápsula 32 Tipo 3

$\begin{array}{llllllllllll}548 & 556 & 557 & 561 & 546 & 551 & 558 & 549 & 530 & 538 & 542 & 541\end{array}$

$\begin{array}{llllllllll}554 & 555 & 547 & 536 & 545 & 560 & 537 & 552 & 533 & 559\end{array}$

$\begin{array}{llllllllll}550 & 543 & 553 & 540 & 535 & 544 & 532 & 531 & 534 & 539\end{array}$

Cápsula 33 Tipo 3

$\begin{array}{llllllllllll}598 & 603 & 604 & 600 & 601 & 599 & 590 & 593 & 596 & 597 & 589 & 592\end{array}$

$\begin{array}{llllllllll}587 & 595 & 591 & 582 & 594 & 605 & 585 & 586 & 583 & 588\end{array}$

$\begin{array}{llllllllll}584 & 602 & 566 & 570 & 563 & 575 & 571 & 569 & 568 & 576\end{array}$

Cápsula 34 Tipo 3

$\begin{array}{llllllllllll}666 & 668 & 671 & 677 & 658 & 650 & 659 & 675 & 656 & 653 & 660 & 655\end{array}$

$\begin{array}{llllllllll}657 & 609 & 654 & 652 & 651 & 608 & 621 & 615 & 610 & 620\end{array}$

$\begin{array}{llllllllll}625 & 612 & 613 & 622 & 618 & 611 & 616 & 617 & 606 & 607\end{array}$

Cápsula 35 Tipo 3

$\begin{array}{llllllllllll}687 & 684 & 685 & 681 & 686 & 680 & 678 & 683 & 679 & 688 & 692 & 689\end{array}$

$\begin{array}{llllllllll}802 & 797 & 808 & 812 & 799 & 800 & 809 & 807 & 1115 & 1114\end{array}$

$\begin{array}{llllllllll}1105 & 1111 & 1106 & 1109 & 1107 & 1110 & 1112 & 1108 & 936 & 1113\end{array}$

Cápsula 36 Tipo 3

$\begin{array}{llllllllllll}1137 & 1139 & 1145 & 1136 & 1146 & 1147 & 1138 & 1135 & 1143 & 1133 & 1123 & 1124\end{array}$

$\begin{array}{llllllllll}1122 & 1121 & 1140 & 1144 & 1141 & 1142 & 1129 & 1132 & 1128 & 1125\end{array}$

$\begin{array}{llllllllll}1131 & 1127 & 1130 & 1126 & 1116 & 1120 & 1119 & 1117 & 1118 & 1134\end{array}$

Cápsula 37 Tipo 3

$\begin{array}{llllllllllll}1164 & 1159 & 1163 & 1160 & 1161 & 1162 & 1151 & 1158 & 1153 & 1154 & 1156 & 1157\end{array}$

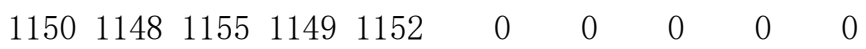

$\begin{array}{llllllllll}0 & 0 & 0 & 0 & 0 & 0 & 0 & 0 & 0 & 0\end{array}$ 
Solución \#2 (3 1 2): Listado de carga del calor residual (decaimiento) en cápsulas MPC-32

Cápsula 1 Tipo 1 Q-A 16.1737 Q-B $\quad 8.3780$ Q-AB 24.5517 Q-M 22.7683 SD-Q 3.001163 $\begin{array}{llllllllllll}1.3740 & 1.3706 & 1.3672 & 1.3638 & 1.3503 & 1.3436 & 1.3436 & 1.3368 & 1.3368 & 1.3335 & 1.3301 & 1.3234\end{array}$ $\begin{array}{llllllllll}0.4189 & 0.4189 & 0.4189 & 0.4189 & 0.4189 & 0.4189 & 0.4189 & 0.4189 & 0.4189 & 0.4189\end{array}$ $\begin{array}{llllllllll}0.4189 & 0.4189 & 0.4189 & 0.4189 & 0.4189 & 0.4189 & 0.4189 & 0.4189 & 0.4189 & 0.4189\end{array}$

Cápsula 2 Tipo 1 Q-A 14.7039 Q-B 8.8630 Q-AB 23.5669 Q-M 22.7683 SD-Q 3.001163 $\begin{array}{llllllllllll}1.2641 & 1.2510 & 1.2478 & 1.2478 & 1.2446 & 1.2124 & 1.2124 & 1.2092 & 1.2060 & 1.2060 & 1.2029 & 1.1997\end{array}$ $\begin{array}{lllllllllll}0.4750 & 0.4750 & 0.4603 & 0.4599 & 0.4585 & 0.4585 & 0.4571 & 0.4571 & 0.4562 & 0.4524\end{array}$ $\begin{array}{lllllllllll}0.4518 & 0.4504 & 0.4368 & 0.4354 & 0.4213 & 0.4196 & 0.4196 & 0.4117 & 0.4089 & 0.3975\end{array}$

Cápsula 3 Tipo 2 Q-A 7.9785 Q-B 8.3780 Q-AB 16.3565 Q-M 22.7683 SD-Q 3.001163 $\begin{array}{llllllllllllll}0.7813 & 0.7673 & 0.6512 & 0.6495 & 0.6477 & 0.6442 & 0.6407 & 0.6407 & 0.6407 & 0.6407 & 0.6390 & 0.6355\end{array}$ $\begin{array}{llllllllllll}0.4189 & 0.4189 & 0.4189 & 0.4189 & 0.4189 & 0.4189 & 0.4189 & 0.4189 & 0.4189 & 0.4189\end{array}$ $\begin{array}{lllllllllll}0.4189 & 0.4189 & 0.4189 & 0.4189 & 0.4189 & 0.4189 & 0.4189 & 0.4189 & 0.4189 & 0.4189\end{array}$

Cápsula 4 Tipo 2 Q-A 8.4150 Q-B $\quad 9.5715$ Q-AB 17.9865 Q-M 22.7683 SD-Q 3.001163 $\begin{array}{lllllllllllll}0.7733 & 0.7260 & 0.7241 & 0.7203 & 0.7199 & 0.7184 & 0.7184 & 0.7117 & 0.6543 & 0.6525 & 0.6507 & 0.6454\end{array}$ $\begin{array}{llllllllllll}0.6080 & 0.5979 & 0.5962 & 0.5841 & 0.5818 & 0.5789 & 0.5789 & 0.4189 & 0.4189 & 0.4189\end{array}$ $\begin{array}{llllllllll}0.4189 & 0.4189 & 0.4189 & 0.4189 & 0.4189 & 0.4189 & 0.4189 & 0.4189 & 0.4189 & 0.4189\end{array}$

Cápsula 5 Tipo 2 Q-A 8.4933 Q-B 11.3368 Q-AB 19.8301 Q-M 22.7683 SD-Q 3.001163 $\begin{array}{lllllllllllll}0.8315 & 0.7146 & 0.7128 & 0.7109 & 0.7090 & 0.7071 & 0.7034 & 0.6904 & 0.6802 & 0.6794 & 0.6783 & 0.6757\end{array}$ $\begin{array}{lllllllllll}0.6098 & 0.6084 & 0.6014 & 0.5835 & 0.5801 & 0.5784 & 0.5766 & 0.5766 & 0.5749 & 0.5732\end{array}$ $\begin{array}{llllllllllll}0.5721 & 0.5704 & 0.5562 & 0.5484 & 0.5477 & 0.5477 & 0.5384 & 0.5318 & 0.5310 & 0.5302\end{array}$

Cápsula 6 Tipo 2 Q-A 11.0235 Q-B 10.8309 Q-AB 21.8544 Q-M 22.7683 SD-Q 3.001163 $\begin{array}{lllllllllllll}0.9883 & 0.9858 & 0.9784 & 0.9784 & 0.9759 & 0.9759 & 0.9735 & 0.9710 & 0.8336 & 0.8233 & 0.8212 & 0.7182\end{array}$ $\begin{array}{lllllllllll}0.6101 & 0.6098 & 0.6098 & 0.6049 & 0.6010 & 0.5962 & 0.5789 & 0.5772 & 0.5772 & 0.5302\end{array}$ $\begin{array}{llllllllll}0.5302 & 0.5302 & 0.5236 & 0.4836 & 0.4836 & 0.4820 & 0.4772 & 0.4756 & 0.4756 & 0.4740\end{array}$

Cápsula 7 Tipo 2 Q-A 9.0536 Q-B 11.6656 Q-AB 20.7192 Q-M 22.7683 SD-Q 3.001163 $\begin{array}{llllllllllll}0.7677 & 0.7658 & 0.7658 & 0.7638 & 0.7638 & 0.7638 & 0.7638 & 0.7483 & 0.7483 & 0.7463 & 0.7323 & 0.7239\end{array}$ $\begin{array}{lllllllllll}0.6296 & 0.6296 & 0.6296 & 0.6010 & 0.5997 & 0.5997 & 0.5979 & 0.5979 & 0.5979 & 0.5962\end{array}$ $\begin{array}{lllllllllll}0.5962 & 0.5962 & 0.5962 & 0.5940 & 0.5927 & 0.5351 & 0.5335 & 0.5335 & 0.5335 & 0.4756\end{array}$

Cápsula 8 Tipo 2 Q-A 9.8574 Q-B 10.8723 Q-AB 20.7297 Q-M 22.7683 SD-Q 3.001163 $\begin{array}{llllllllllllll}0.9063 & 0.8908 & 0.8886 & 0.8820 & 0.8688 & 0.8666 & 0.7776 & 0.7680 & 0.7620 & 0.7502 & 0.7502 & 0.7463\end{array}$ $\begin{array}{llllllllllll}0.6183 & 0.6165 & 0.6147 & 0.6112 & 0.5875 & 0.5858 & 0.5853 & 0.5451 & 0.5451 & 0.5401\end{array}$ $\begin{array}{llllllllll}0.5343 & 0.5335 & 0.4974 & 0.4974 & 0.4958 & 0.4942 & 0.4942 & 0.4925 & 0.4925 & 0.4909\end{array}$

Cápsula 9 Tipo 2 Q-A 9.4775 Q-B 10.9381 Q-AB 20.4156 Q-M 22.7683 SD-Q 3.001163 $\begin{array}{llllllllllllll}0.8514 & 0.8107 & 0.8066 & 0.8046 & 0.7923 & 0.7882 & 0.7781 & 0.7724 & 0.7704 & 0.7704 & 0.7680 & 0.7644\end{array}$ $\begin{array}{lllllllllll}0.5910 & 0.5905 & 0.5858 & 0.5841 & 0.5813 & 0.5789 & 0.5444 & 0.5434 & 0.5434 & 0.5418\end{array}$ $\begin{array}{llllllllllll}0.5418 & 0.5351 & 0.5335 & 0.5327 & 0.5318 & 0.5302 & 0.5302 & 0.5088 & 0.5055 & 0.5039\end{array}$

Cápsula 10 Tipo 2 Q-A 12.4668 Q-B 11.0123 Q-AB 23.4791 Q-M 22.7683 SD-Q 3.001163 $\begin{array}{lllllllllllll}1.1389 & 1.1306 & 1.1278 & 1.1223 & 1.0027 & 1.0027 & 0.9955 & 0.9955 & 0.9931 & 0.9907 & 0.9835 & 0.9835\end{array}$ $\begin{array}{llllllllllll}0.6094 & 0.5990 & 0.5973 & 0.5973 & 0.5848 & 0.5844 & 0.5831 & 0.5533 & 0.5500 & 0.5467\end{array}$ $\begin{array}{llllllllllll}0.5417 & 0.5417 & 0.5270 & 0.5270 & 0.5253 & 0.5253 & 0.5072 & 0.5064 & 0.5031 & 0.5023\end{array}$

Cápsula 11 Tipo 2 Q-A 12.1437 Q-B 11.4446 Q-AB 23.5883 Q-M 22.7683 SD-Q 3.001163 $\begin{array}{llllllllllllll}1.0226 & 1.0201 & 1.0201 & 1.0176 & 1.0151 & 1.0126 & 1.0126 & 1.0076 & 1.0076 & 1.0026 & 1.0026 & 1.0026\end{array}$ $\begin{array}{lllllllllll}0.6172 & 0.6154 & 0.6154 & 0.6119 & 0.6101 & 0.6025 & 0.6007 & 0.5973 & 0.5939 & 0.5550\end{array}$ $\begin{array}{llllllllllll}0.5533 & 0.5533 & 0.5500 & 0.5484 & 0.5477 & 0.5394 & 0.5384 & 0.5360 & 0.5351 & 0.5236\end{array}$

Cápsula 12 Tipo 2 Q-A 12.7274 Q-B 11.6941 Q-AB 24.4215 Q-M 22.7683 SD-Q 3.001163 $\begin{array}{lllllllllllll}1.1306 & 1.1196 & 1.1168 & 1.1141 & 1.0868 & 1.0814 & 1.0652 & 1.0439 & 0.9994 & 0.9916 & 0.9890 & 0.9890\end{array}$ $\begin{array}{llllllllllll}0.6303 & 0.6285 & 0.6233 & 0.6233 & 0.6199 & 0.6165 & 0.6154 & 0.6137 & 0.6101 & 0.6079\end{array}$ $\begin{array}{llllllllllll}0.6028 & 0.5977 & 0.5494 & 0.5477 & 0.5410 & 0.5368 & 0.5360 & 0.5351 & 0.5351 & 0.5236\end{array}$ 
Cápsula 13 Tipo 2 Q-A 12.6180 Q-B 11.7133 Q-AB 24.3313 Q-M 22.7683 SD-Q 3.001163

$\begin{array}{lllllllllllll}1.0733 & 1.0733 & 1.0733 & 1.0679 & 1.0652 & 1.0519 & 1.0439 & 1.0412 & 1.0412 & 1.0333 & 1.0281 & 1.0254\end{array}$

$\begin{array}{llllllllll}0.6289 & 0.6285 & 0.6279 & 0.6261 & 0.6261 & 0.6251 & 0.6244 & 0.6244 & 0.6218 & 0.6216\end{array}$

$\begin{array}{lllllllllll}0.6199 & 0.6079 & 0.6062 & 0.6045 & 0.6045 & 0.6045 & 0.4566 & 0.4535 & 0.4520 & 0.4489\end{array}$

Cápsula 14 Tipo 2 Q-A 13.5842 Q-B 9.8348 Q-AB 23.4190 Q-M 22.7683 SD-Q 3.001163 $\begin{array}{lllllllllllll}1.2575 & 1.2543 & 1.2381 & 1.1278 & 1.1278 & 1.1196 & 1.1168 & 1.0760 & 1.0706 & 1.0679 & 1.0679 & 1.0599\end{array}$ $\begin{array}{llllllllll}0.6307 & 0.5947 & 0.5947 & 0.5923 & 0.5923 & 0.5375 & 0.5207 & 0.5207 & 0.5186 & 0.5159\end{array}$ $\begin{array}{llllllllll}0.5123 & 0.4785 & 0.4292 & 0.4069 & 0.4038 & 0.4022 & 0.3975 & 0.3975 & 0.3975 & 0.3913\end{array}$

Cápsula 15 Tipo 2 Q-A 14.0575 Q-B $\quad 9.8885$ Q-AB 23.9460 Q-M 22.7683 SD-Q 3.001163 $\begin{array}{llllllllllll}1.2543 & 1.2543 & 1.2510 & 1.2502 & 1.2380 & 1.2336 & 1.1614 & 1.1614 & 1.0706 & 1.0652 & 1.0603 & 1.0572\end{array}$ $\begin{array}{llllllllll}0.5477 & 0.5426 & 0.5426 & 0.5375 & 0.5273 & 0.5240 & 0.5234 & 0.5223 & 0.5223 & 0.5156\end{array}$ $\begin{array}{llllllllll}0.5133 & 0.4836 & 0.4651 & 0.4651 & 0.4651 & 0.4635 & 0.4603 & 0.4372 & 0.4325 & 0.3975\end{array}$

Cápsula 16 Tipo 2 Q-A 14.1221 Q-B 9.9201 Q-AB 24.0422 Q-M 22.7683 SD-Q 3.001163 $\begin{array}{lllllllllllll}1.2428 & 1.2367 & 1.2367 & 1.2367 & 1.2306 & 1.2306 & 1.1585 & 1.1555 & 1.1526 & 1.1496 & 1.1485 & 0.9433\end{array}$ $\begin{array}{lllllllllll}0.5477 & 0.5460 & 0.5409 & 0.5240 & 0.5223 & 0.5206 & 0.5206 & 0.5190 & 0.5173 & 0.5140\end{array}$ $\begin{array}{lllllllllll}0.5140 & 0.5140 & 0.4667 & 0.4619 & 0.4603 & 0.4587 & 0.4555 & 0.4539 & 0.4492 & 0.4135\end{array}$

Cápsula 17 Tipo 2 Q-A 12.0803 Q-B 10.0341 Q-AB 22.1144 Q-M 22.7683 SD-Q 3.001163 $\begin{array}{llllllllllllll}1.1233 & 1.1204 & 1.1175 & 1.1146 & 0.9762 & 0.9584 & 0.9534 & 0.9508 & 0.9458 & 0.9408 & 0.9408 & 0.9383\end{array}$ $\begin{array}{lllllllllll}0.6114 & 0.5576 & 0.5558 & 0.5507 & 0.5455 & 0.5167 & 0.5167 & 0.5015 & 0.4934 & 0.4902\end{array}$ $\begin{array}{llllllllll}0.4885 & 0.4820 & 0.4820 & 0.4787 & 0.4771 & 0.4755 & 0.4656 & 0.4594 & 0.4546 & 0.4312\end{array}$

Cápsula 18 Tipo 2 Q-A 12.1664 Q-B $\quad 9.6970$ Q-AB 21.8634 Q-M 22.7683 SD-Q 3.001163 $\begin{array}{llllllllllllll}1.0859 & 1.0830 & 1.0802 & 1.0716 & 1.0631 & 0.9813 & 0.9788 & 0.9762 & 0.9660 & 0.9635 & 0.9609 & 0.9559\end{array}$ $\begin{array}{lllllllllll}0.6120 & 0.5477 & 0.5257 & 0.5210 & 0.5190 & 0.5012 & 0.5012 & 0.4744 & 0.4731 & 0.4700\end{array}$ $\begin{array}{lllllllllll}0.4683 & 0.4656 & 0.4656 & 0.4651 & 0.4635 & 0.4554 & 0.4554 & 0.4476 & 0.4354 & 0.4298\end{array}$

Cápsula 19 Tipo 2 Q-A 12.2284 Q-B 9.4969 Q-AB 21.7253 Q-M 22.7683 SD-Q 3.001163 $\begin{array}{llllllllllllll}1.0660 & 1.0631 & 1.0575 & 1.0547 & 1.0547 & 1.0547 & 0.9839 & 0.9813 & 0.9813 & 0.9788 & 0.9762 & 0.9762\end{array}$ $\begin{array}{llllllllllll}0.5256 & 0.5179 & 0.5179 & 0.5148 & 0.5148 & 0.5133 & 0.5027 & 0.5012 & 0.4729 & 0.4714\end{array}$ $\begin{array}{lllllllllll}0.4656 & 0.4641 & 0.4583 & 0.4439 & 0.4397 & 0.4368 & 0.4368 & 0.4354 & 0.4326 & 0.4312\end{array}$

Cápsula 20 Tipo 2 Q-A 12.2158 Q-B 10.1992 Q-AB 22.4150 Q-M 22.7683 SD-Q 3.001163 $\begin{array}{llllllllllllll}1.0830 & 1.0631 & 1.0603 & 1.0575 & 1.0547 & 1.0491 & 0.9890 & 0.9788 & 0.9775 & 0.9721 & 0.9667 & 0.9640\end{array}$ $\begin{array}{lllllllllll}0.5957 & 0.5925 & 0.5318 & 0.5302 & 0.5287 & 0.5256 & 0.5240 & 0.5042 & 0.5033 & 0.5019\end{array}$ $\begin{array}{lllllllllll}0.5019 & 0.5012 & 0.4997 & 0.4997 & 0.4945 & 0.4931 & 0.4901 & 0.4887 & 0.4598 & 0.4326\end{array}$

Cápsula 21 Tipo 2 Q-A 13.2246 Q-B 10.7288 Q-AB 23.9534 Q-M 22.7683 SD-Q 3.001163 $\begin{array}{lllllllllllll}1.2543 & 1.2510 & 1.0859 & 1.0773 & 1.0745 & 1.0745 & 1.0716 & 1.0716 & 1.0688 & 1.0688 & 1.0660 & 1.0603\end{array}$ $\begin{array}{llllllllll}0.6053 & 0.6021 & 0.6005 & 0.5989 & 0.5989 & 0.5973 & 0.5470 & 0.5424 & 0.5348 & 0.5122\end{array}$ $\begin{array}{lllllllllll}0.5078 & 0.5063 & 0.5048 & 0.5033 & 0.4989 & 0.4975 & 0.4960 & 0.4960 & 0.4901 & 0.4887\end{array}$

Cápsula 22 Tipo 2 Q-A 13.9198 Q-B 10.2035 Q-AB 24.1233 Q-M 22.7683 SD-Q 3.001163 $\begin{array}{lllllllllllll}1.2608 & 1.2608 & 1.2575 & 1.2543 & 1.2510 & 1.0973 & 1.0973 & 1.0944 & 1.0916 & 1.0916 & 1.0887 & 1.0745\end{array}$ $\begin{array}{lllllllllll}0.5713 & 0.5713 & 0.5409 & 0.5409 & 0.5394 & 0.5394 & 0.5363 & 0.5237 & 0.5152 & 0.5152\end{array}$ $\begin{array}{lllllllllll}0.5122 & 0.5078 & 0.5078 & 0.5048 & 0.4706 & 0.4628 & 0.4628 & 0.4613 & 0.4599 & 0.4599\end{array}$

Cápsula 23 Tipo 2 Q-A 13.5046 Q-B 9.6953 Q-AB 23.1999 Q-M 22.7683 SD-Q 3.001163 $\begin{array}{lllllllllllll}1.2608 & 1.1556 & 1.1185 & 1.1185 & 1.1154 & 1.1124 & 1.1124 & 1.1093 & 1.1093 & 1.1093 & 1.0944 & 1.0887\end{array}$ $\begin{array}{lllllllllll}0.5713 & 0.5681 & 0.5382 & 0.5382 & 0.5305 & 0.4794 & 0.4735 & 0.4720 & 0.4706 & 0.4691\end{array}$ $\begin{array}{llllllllll}0.4662 & 0.4633 & 0.4619 & 0.4619 & 0.4590 & 0.4576 & 0.4576 & 0.4547 & 0.4532 & 0.4490\end{array}$

Cápsula 24 Tipo 2 Q-A 14.3787 Q-B 10.0612 Q-AB 24.4399 Q-M 22.7683 SD-Q 3.001163 $\begin{array}{lllllllllllll}1.2575 & 1.2510 & 1.2349 & 1.2317 & 1.2284 & 1.2252 & 1.2220 & 1.2124 & 1.1587 & 1.1556 & 1.1494 & 1.0519\end{array}$ $\begin{array}{lllllllllll}0.5618 & 0.5602 & 0.5554 & 0.5539 & 0.5351 & 0.5336 & 0.5336 & 0.5336 & 0.5321 & 0.5290\end{array}$ $\begin{array}{lllllllllll}0.5290 & 0.4677 & 0.4648 & 0.4604 & 0.4561 & 0.4561 & 0.4518 & 0.4490 & 0.4490 & 0.4490\end{array}$

Cápsula 25 Tipo 2 Q-A 12.9583 Q-B 7.2928 Q-AB 20.2511 Q-M 22.7683 SD-Q 3.001163 $\begin{array}{lllllllllllll}1.2575 & 1.2543 & 1.2381 & 1.2349 & 1.2349 & 1.2156 & 1.2092 & 1.0639 & 1.0639 & 1.0609 & 0.5649 & 0.5602\end{array}$ $\begin{array}{lllllllllll}0.5554 & 0.5539 & 0.5398 & 0.5336 & 0.4764 & 0.4750 & 0.4720 & 0.4706 & 0.4677 & 0.4677\end{array}$ $\begin{array}{llllllllllll}0.4677 & 0.4604 & 0.4547 & 0.4518 & 0.4461 & 0.0000 & 0.0000 & 0.0000 & 0.0000 & 0.0000\end{array}$ 
Cápsula 26 Tipo 3 Q-A 8.4949 Q-B 12.9675 Q-AB 21.4624 Q-M 22.7683 SD-Q 3.001163

$\begin{array}{llllllllllllll}0.7593 & 0.7442 & 0.7112 & 0.7105 & 0.7068 & 0.7052 & 0.6973 & 0.6955 & 0.6917 & 0.6917 & 0.6917 & 0.6898\end{array}$

$\begin{array}{lllllllllll}0.6898 & 0.6860 & 0.6571 & 0.6571 & 0.6553 & 0.6535 & 0.6512 & 0.6477 & 0.6460 & 0.6442\end{array}$

$\begin{array}{llllllllll}0.6442 & 0.6425 & 0.6407 & 0.6390 & 0.6385 & 0.6363 & 0.6355 & 0.6350 & 0.6347 & 0.6332\end{array}$

Cápsula 27 Tipo 3 Q-A 8.6284 Q-B 12.8145 Q-AB 21.4429 Q-M 22.7683 SD-Q 3.001163

$\begin{array}{lllllllllllll}0.7793 & 0.7753 & 0.7713 & 0.7238 & 0.7184 & 0.7180 & 0.7165 & 0.7117 & 0.7097 & 0.6923 & 0.6578 & 0.6543\end{array}$

$\begin{array}{llllllllllll}0.6525 & 0.6525 & 0.6507 & 0.6490 & 0.6490 & 0.6472 & 0.6460 & 0.6454 & 0.6442 & 0.6372\end{array}$

$\begin{array}{lllllllllll}0.6372 & 0.6372 & 0.6355 & 0.6355 & 0.6337 & 0.6337 & 0.6320 & 0.6320 & 0.6320 & 0.6320\end{array}$

Cápsula 28 Tipo 3 Q-A 9.2238 Q-B 13.8621 Q-AB 23.0859 Q-M 22.7683 SD-Q 3.001163

$\begin{array}{llllllllllllll}0.8482 & 0.8357 & 0.8336 & 0.8315 & 0.8274 & 0.7258 & 0.7239 & 0.7222 & 0.7203 & 0.7203 & 0.7184 & 0.7165\end{array}$

$\begin{array}{lllllllllll}0.7165 & 0.7146 & 0.7128 & 0.7109 & 0.7071 & 0.7071 & 0.6991 & 0.6972 & 0.6923 & 0.6915\end{array}$

$\begin{array}{llllllllll}0.6915 & 0.6904 & 0.6839 & 0.6839 & 0.6794 & 0.6775 & 0.6775 & 0.6775 & 0.6757 & 0.6757\end{array}$

Cápsula 29 Tipo 3 Q-A 9.6293 Q-B 15.0268 Q-AB 24.6561 Q-M 22.7683 SD-Q 3.001163

$\begin{array}{lllllllllllllll}0.8623 & 0.8558 & 0.8441 & 0.8357 & 0.8315 & 0.7795 & 0.7736 & 0.7720 & 0.7717 & 0.7677 & 0.7677 & 0.7677\end{array}$

$\begin{array}{lllllllllll}0.7658 & 0.7640 & 0.7640 & 0.7640 & 0.7638 & 0.7638 & 0.7599 & 0.7560 & 0.7560 & 0.7540\end{array}$

$\begin{array}{lllllllllll}0.7521 & 0.7502 & 0.7483 & 0.7463 & 0.7463 & 0.7406 & 0.7386 & 0.7343 & 0.7304 & 0.7284\end{array}$

Cápsula 30 Tipo 3 Q-A 10.4143 Q-B 15.6578 Q-AB 26.0721 Q-M 22.7683 SD-Q 3.001163

$\begin{array}{lllllllllllllll}0.8952 & 0.8908 & 0.8886 & 0.8864 & 0.8842 & 0.8710 & 0.8623 & 0.8601 & 0.8579 & 0.8558 & 0.8492 & 0.8128\end{array}$

$\begin{array}{llllllllllll}0.7943 & 0.7943 & 0.7923 & 0.7903 & 0.7903 & 0.7903 & 0.7903 & 0.7903 & 0.7882 & 0.7882\end{array}$

$\begin{array}{lllllllllll}0.7842 & 0.7842 & 0.7781 & 0.7761 & 0.7744 & 0.7720 & 0.7720 & 0.7700 & 0.7700 & 0.7680\end{array}$

Cápsula 31 Tipo 3 Q-A 10.9544 Q-B 16.2147 Q-AB 27.1691 Q-M 22.7683 SD-Q 3.001163

$\begin{array}{llllllllllllll}0.9295 & 0.9249 & 0.9203 & 0.9180 & 0.9180 & 0.9157 & 0.9157 & 0.9134 & 0.9111 & 0.8997 & 0.8974 & 0.8907\end{array}$

$\begin{array}{llllllllll}0.8367 & 0.8345 & 0.8345 & 0.8303 & 0.8256 & 0.8213 & 0.8192 & 0.8149 & 0.8134 & 0.8128\end{array}$

$\begin{array}{llllllllll}0.8087 & 0.8086 & 0.8046 & 0.8030 & 0.8030 & 0.7968 & 0.7903 & 0.7882 & 0.7882 & 0.7801\end{array}$

Cápsula 32 Tipo 3 Q-A 10.0594 Q-B 16.3543 Q-AB 26.4137 Q-M 22.7683 SD-Q 3.001163

$\begin{array}{lllllllllllllll}0.8527 & 0.8483 & 0.8460 & 0.8460 & 0.8439 & 0.8395 & 0.8373 & 0.8351 & 0.8303 & 0.8303 & 0.8261 & 0.8239\end{array}$

$\begin{array}{lllllllllll}0.8218 & 0.8218 & 0.8218 & 0.8218 & 0.8218 & 0.8218 & 0.8218 & 0.8197 & 0.8197 & 0.8197\end{array}$

$\begin{array}{lllllllllll}0.8197 & 0.8197 & 0.8155 & 0.8155 & 0.8155 & 0.8134 & 0.8134 & 0.8113 & 0.8093 & 0.8093\end{array}$

Cápsula 33 Tipo 3 Q-A 10.4617 Q-B 16.3972 Q-AB 26.8589 Q-M 22.7683 SD-Q 3.001163

$\begin{array}{lllllllllllllll}0.8929 & 0.8929 & 0.8861 & 0.8839 & 0.8839 & 0.8816 & 0.8615 & 0.8571 & 0.8571 & 0.8571 & 0.8549 & 0.8527\end{array}$

$\begin{array}{llllllllllll}0.8505 & 0.8505 & 0.8483 & 0.8483 & 0.8460 & 0.8460 & 0.8417 & 0.8395 & 0.8329 & 0.8329\end{array}$

$\begin{array}{lllllllllll}0.8329 & 0.8285 & 0.7989 & 0.7923 & 0.7901 & 0.7901 & 0.7879 & 0.7836 & 0.7814 & 0.7749\end{array}$

Cápsula 34 Tipo 3 Q-A 10.8005 Q-B 17.4282 Q-AB 28.2287 Q-M 22.7683 SD-Q 3.001163

$\begin{array}{lllllllllllll}0.9106 & 0.9083 & 0.9083 & 0.9059 & 0.9036 & 0.8965 & 0.8965 & 0.8965 & 0.8965 & 0.8942 & 0.8918 & 0.8918\end{array}$

$\begin{array}{lllllllllll}0.8918 & 0.8907 & 0.8895 & 0.8895 & 0.8872 & 0.8839 & 0.8794 & 0.8771 & 0.8771 & 0.8749\end{array}$

$\begin{array}{lllllllllll}0.8749 & 0.8727 & 0.8727 & 0.8704 & 0.8615 & 0.8549 & 0.8549 & 0.8549 & 0.8351 & 0.8351\end{array}$

Cápsula 35 Tipo 3 Q-A 10.8853 Q-B 14.7054 Q-AB 25.5907 Q-M 22.7683 SD-Q 3.001163

$\begin{array}{llllllllllllll}0.9201 & 0.9177 & 0.9130 & 0.9130 & 0.9059 & 0.9059 & 0.9036 & 0.9036 & 0.9012 & 0.9012 & 0.9012 & 0.8989\end{array}$

$\begin{array}{lllllllllll}0.8826 & 0.8749 & 0.8749 & 0.8749 & 0.8724 & 0.8698 & 0.8673 & 0.8673 & 0.6565 & 0.6530\end{array}$

$\begin{array}{lllllllllll}0.6512 & 0.6477 & 0.6460 & 0.6407 & 0.6407 & 0.6390 & 0.6390 & 0.6372 & 0.6366 & 0.6337\end{array}$

Cápsula 36 Tipo 3 Q-A 8.5525 Q-B 13.3555 Q-AB 21.9080 Q-M 22.7683 SD-Q 3.001163

$\begin{array}{llllllllllllll}0.7222 & 0.7203 & 0.7203 & 0.7184 & 0.7165 & 0.7165 & 0.7146 & 0.7146 & 0.7146 & 0.7097 & 0.6924 & 0.6924\end{array}$

$\begin{array}{llllllllllll}0.6924 & 0.6905 & 0.6794 & 0.6789 & 0.6739 & 0.6720 & 0.6708 & 0.6708 & 0.6690 & 0.6690\end{array}$

$\begin{array}{lllllllllll}0.6690 & 0.6672 & 0.6672 & 0.6654 & 0.6601 & 0.6601 & 0.6548 & 0.6548 & 0.6495 & 0.6407\end{array}$

Cápsula 37 Tipo 3 Q-A $\quad 8.8231$ Q-B $\quad 3.3930$ Q-AB 12.2161 Q-M 22.7683 SD-Q 3.001163

$\begin{array}{llllllllllllll}0.7713 & 0.7593 & 0.7534 & 0.7494 & 0.7475 & 0.7279 & 0.7260 & 0.7260 & 0.7203 & 0.7184 & 0.7146 & 0.7090\end{array}$

$\begin{array}{llllllllll}0.7053 & 0.6739 & 0.6734 & 0.6720 & 0.6684 & 0.0000 & 0.0000 & 0.0000 & 0.0000 & 0.0000\end{array}$

$\begin{array}{llllllllllll}0.0000 & 0.0000 & 0.0000 & 0.0000 & 0.0000 & 0.0000 & 0.0000 & 0.0000 & 0.0000 & 0.0000\end{array}$ 